\title{
New Geometric Flows on Riemannian Manifolds and Applications to Schrödinger-Airy Flows
}

\author{
Xiaowei Sun*and Youde Wang ${ }^{\dagger}$
}

\begin{abstract}
In this paper, we define a class of new geometric flows on a complete Riemannian manifold. The new flow is related to the generalized (third order) Landau-Lifishitz equation. On the other hand it could be thought of a special case of the Schrödinger-Airy flow when the target manifold is a Kähler manifold with constant holomorphic sectional curvature. We show the local existence of the new flow on a complete Riemannian manifold with some assumptions on Ricci tensor. Moreover, if the target manifolds are Einstein or some certain type of locally symmetric spaces, we obtain the global results.
\end{abstract}

\section{Introduction}

Let $(N, h)$ be a Riemannian manifold equipped with a metric $h$. Then there is a Levi-Civita connection associated to $h$. Denote the Levi-Civita connection on $(N, h)$ by $\nabla$ and the corresponding curvature operator on $(N, h)$ by $R$, which is a $(1,3)$-tensor.

The Ricci curvature Ric is the trace of the curvature operator $R$. It is well-known that Ric is a symmetric bilinear form. If for any tangent vector fields $X, Y$ on $(N, h)$ there holds

$$
\operatorname{Ric}(X, Y)=k h(X, Y),
$$

then $(N, h)$ is said to be an Einstein manifold with Einstein constant $k$.

For any smooth map $u(x, t)$ from $S^{1} \times \mathbb{R}$ into $(N, h)$, Let $\nabla_{x}$ denote the covariant derivative $\nabla_{\frac{\partial}{\partial x}}$ on the pull-back bundle $u^{-1} T N$ induced from the Levi-Civita connection $\nabla$ on $N$. For the sake of convenience, we always denote $\nabla_{x} u$ and $\nabla_{t} u$ by $u_{x}$ and $u_{t}$ respectively.

For the maps from a unit circle $S^{1}$ or a real line $\mathbb{R}$ into $N$, we define a class of new geometric flows as follows:

$$
\frac{\partial u}{\partial t}=\nabla_{x}^{2} u_{x}+\rho \operatorname{Ric}\left(u_{x}, u_{x}\right) u_{x}
$$

where $\rho$ is a positive constant. If $(N, h)$ is an Einstein manifold, the new geometric flow is an energy conserved system.

\footnotetext{
${ }^{*}$ Supported by NSFC, Grant No. 11226082

${ }^{\dagger}$ Supported by NSFC, Grant No. 10990013
} 
Obviously, if the target manifold is Euclidian space $\mathbb{R}^{n}$, the new flow then reduces to the vector Airy equation

$$
u_{t}=u_{x x x}
$$

We should mention that solutions to the scalar Airy equation may be expressed in terms of so-called Airy functions, which are named after the astronomer George Biddell Airy(1801-1892) and are solutions to the differential equation

$$
y_{x x}(x)-x y(x)=0 .
$$

One of the related problems with our new flow is the so-called generalized Landau-Lifishitz equation written by

$$
u_{t}=u_{x x x}+\frac{3}{2}\left(\left|u_{x}\right|^{2} u\right)_{x}, \quad|u|^{2}=1
$$

Here $u: S^{1}($ or $\mathbb{R}) \times \mathbb{R} \rightarrow S^{n} \subset \mathbb{R}^{n+1}$ and $|\cdot|$ denotes the standard metric in $\mathbb{R}^{n+1}$ (see $[4,17,30])$. If $n=2$, it is just the third order Landau-Lifshitz equation. It is easy to see that this equation can be rewritten by a geometric version as follows:

$$
u_{t}=\nabla_{x}^{2} u_{x}+\frac{1}{2}\left|u_{x}\right|^{2} u_{x}
$$

where $\nabla_{x}=\nabla_{\frac{\partial}{\partial x}}$ denotes the Levi-Civita connection on the standard unit sphere $S^{n}$. For detail we refer to [26]. Furthermore, we may intrinsically write the equation by

$$
u_{t}=\nabla_{x}^{2} u_{x}+\frac{1}{2(n-1)} \operatorname{Ric}\left(u_{x}, u_{x}\right) u_{x}
$$

where $\operatorname{Ric}(\cdot, \cdot)$ is the Ricci tensor (operator) on $S^{n}$.

In fact, from the integrable system point of view the following generalized Landau-Lifshitz equation was considered in $[12,17,29]$

$$
u_{t}=\nabla_{x}^{2} u_{x}+\frac{1}{2}\left|u_{x}\right|^{2} u_{x}+\frac{3}{2}\langle u, A(u)\rangle u_{x}
$$

where $u: \mathbb{R} \times \mathbb{R} \rightarrow S^{n} \subset \mathbb{R}^{n+1}$ and $A$ is a constant symmetric $(n+1) \times(n+1)$ matrix. It was shown that this equation is integrable by the inverse scattering method for any $n$ and $A$. One has known that this equation also defines an infinitesimal symmetry for the well-known C. Neumann system $[17,32]$

$$
u_{x x}+\left|u_{x}\right|^{2} u=A(u)-\langle u, A(u)\rangle u, \quad|u|^{2}=1
$$

describing the dynamics of a particle on the unit sphere under the influence of field with the quadratic potential

$$
P=\frac{1}{2}\langle u, A(u)\rangle .
$$

Recently, Song and Yu in [26] employed the geometric energy method established in [7,8] to show the global well-posedness of the corresponding Cauchy problem from $S^{1} \times \mathbb{R} \rightarrow S^{n}$. We 
also mention that the stationary solution of the geometric flow defines an interesting kind of curves on $S^{n}$ (see [25]).

On the other hand, if $(N, J, h)$ is a Kähler manifold, where $J$ is a compatible complex structure with $h$, in [28] we introduced a class of geometric flows for the maps from a unit circle $S^{1}$ or a real line $\mathbb{R}$ into $(N, J, h)$, geometric Schrödinger-Airy flow, as follows:

$$
\frac{\partial u}{\partial t}=\alpha\left(\nabla_{x}^{2} u_{x}+\frac{1}{2} R\left(u_{x}, J_{u} u_{x}\right) J_{u} u_{x}\right)+\beta J_{u} \nabla_{x} u_{x}+\gamma\left|u_{x}\right|^{2} u_{x}
$$

where $\alpha, \beta$ and $\gamma$ are real constants, $R$ is the Riemannian curvature tensor on $N$ and $J_{u} \equiv J(u)$. In [28] we have shown that the Schrödinger-Airy flows relate closely to several important and well-known physical or mechanical systems $[10,11,12,13,15,16,18,19,21,23]$. This flow is hybrid of geometric KdV flow [5, 27] and geometric Schrödinger flow [1, 2, 3, 6, 7, 24], and relates closely to derivative nonlinear Schrödinger equation[28, 33].

If $\beta=0$, the Schrödinger-Airy flow on a Kähler manifold with constant holomorphic sectional curvature $K$ becomes

$$
\frac{\partial u}{\partial t}=\alpha\left(\nabla_{x}^{2} u_{x}+\frac{1}{2} K\left|u_{x}\right|^{2} u_{x}\right)+\gamma\left|u_{x}\right|^{2} u_{x}
$$

By scaling with respect to the time variable, we can change the above flow into

$$
\frac{\partial u}{\partial t}=\nabla_{x}^{2} u_{x}+\rho\left|u_{x}\right|^{2} u_{x}
$$

This is just our new geometric flow on a Kähler manifold with constant holomorphic sectional curvature. However, by our knowledge one has not obtained any global existence results for (1.3). Thus, by choosing $N$ specially, the new flow could be regarded as special cases of the Schrödinger-Airy flow. But in general cases, these two flows differ a lot since the new geometric flow is defined on all Riemannian manifolds while the Schrödinger-Airy flow is only defined on Kähler manifolds.

In this paper, we mainly discuss the local existence for the Cauchy problem of the new geometric flow on a complete Riemannian manifold $(N, h)$ defined by

$$
\left\{\begin{array}{l}
u_{t}=\nabla_{x}^{2} u_{x}+\rho \operatorname{Ric}\left(u_{x}, u_{x}\right) u_{x}, \quad x \in S^{1} ; \\
u(x, 0)=u_{0}(x) .
\end{array}\right.
$$

Furthermore, when $N$ is some kind of special locally symmetric spaces, we could obtain some results on global existence of (1.4). The method we use here is the geometric energy method which is also adopted to discuss the $\mathrm{KdV}$ geometric flow in [27]. But technically speaking, the processes differ greatly especially in proving the existence of the two geometric flows. By utilizing the Ricci curvature tensor, we introduce a new geometric norm which would help us to obtain the estimates we need.

Before stating our main results, we need to introduce several definitions on Sobolev spaces of sections with vector bundle value on $M$. Let $(E, M, \pi)$ be a vector bundle with base manifold $M$. If $(E, M, \pi)$ is equipped with a metric, then we may define so-called vector bundle value Sobolev spaces as follows: 
Definition 1.1. $H^{m}(M, E)$ is the completeness of the set of smooth sections with compact supports denoted by $\left\{s \mid s \in C_{0}^{\infty}(M, E)\right\}$ with respect to the norm

$$
\|s\|_{H^{m}(M, E)}^{2}=\sum_{i=0}^{m} \int_{M}\left|\nabla^{i} s\right|^{2} d M .
$$

Here $\nabla$ is the connection on $E$ which is compatible with the metric on $E$.

Definition 1.2. Let $\mathbb{N}$ be the set of positive integers. For $m \in \mathbb{N} \cup\{0\}$, the Sobolev space of maps from $S^{1}$ into a Riemannian manifold $(N, h)$ is defined by

$$
H^{m+1}\left(S^{1} ; N\right)=\left\{u \in C\left(S^{1} ; N\right) \mid u_{x} \in H^{m}\left(S^{1} ; T N\right)\right\},
$$

where $u_{x} \in H^{m}\left(S^{1} ; T N\right)$ means that $u_{x}$ satisfies

$$
\left\|u_{x}\right\|_{H^{m}\left(S^{1} ; T N\right)}^{2}=\sum_{j=0}^{m} \int_{S^{1}} h(u(x))\left(\nabla_{x}^{j} u_{x}(x), \nabla_{x}^{j} u_{x}(x)\right) d x<+\infty .
$$

We usually use $W^{k, p}(M, N)$ to denote the space of Sobolev maps from $M$ into $N$, and $W^{k, p}\left(M, \mathbb{R}^{l}\right)$ to denote the space of Sobolev functions.

Our main results are as follows:

Theorem 1.1. Let $(N, h)$ be a complete Riemannian manifold with parallel Ricci tensor, i.e. $\nabla$ Ric $\equiv 0$. If the Ricci curvature on $N$ has a positive lower bound $\lambda>0$ (or a negative upper bound $-\lambda<0)$, then the local solutions $u \in L^{\infty}\left([0, T], H^{k}\left(S^{1}, N\right)\right)(k \geq 4)$ of the Cauchy problem (1.4) with the initial map $u_{0} \in H^{k}\left(S^{1}, N\right)$ is unique. Moreover, the local solution is continuous with respect to the time variable, i.e., $u \in C\left([0, T], H^{k}\left(S^{1}, N\right)\right)$.

Theorem 1.2. Let $(N, h)$ be a complete Riemannian manifold with parallel Ricci tensor, i.e. $\nabla R i c \equiv 0$ and the Ricci curvature on $N$ has a positive lower bound $\lambda>0$ (or a negative upper bound $-\lambda<0)$. Then, for any integer $k \geq 4$ the Cauchy problem of (1.4) with the initial value map $u_{0} \in H^{k}\left(S^{1}, N\right)$ admits a unique local solution $u \in C\left([0, T], H^{k}\left(S^{1}, N\right)\right)$, where $T=T\left(N,\left\|u_{0}\right\|_{H^{4}}\right)$. Moreover, besides the assumptions on $N$, if the Riemmainan curvature on $N$ satisfies $\left|\nabla^{l} R\right| \leq B_{l}(l=0,1,2,3)$ where $B_{l}$ are positive constants, then if the initial value map $u_{0} \in H^{3}\left(S^{1}, N\right)$, the Cauchy problem of (1.4) admits a local solution $u \in L^{\infty}\left([0, T], H^{3}\left(S^{1}, N\right)\right)$, where $T=T\left(N,\left\|u_{0}\right\|_{H^{3}}\right)$.

Theorem 1.3. Assume that $(N, h)$ is a complete locally symmetric space on which the Ricci curvature has a positive lower bound $\lambda>0$ (or a negative upper bound $-\lambda<0$ ). Then for any integer $k \geq 4$ the Cauchy problem (1.4) with the initial map $u_{0} \in H^{k}\left(S^{1}, N\right)$ admits a unique global solution $u \in C\left([0, \infty), H^{k}\left(S^{1}, N\right)\right)$.

It is worthy to point out that in this work, we still employ the parabolic approximation and the geometric energy method developed in $[7,8]$ to show the local existence problems. The 
process is similar but different with that about the $\mathrm{KdV}$ geometric flow in [27]. In fact, to show the local existence, we first obtain a local solution $u_{\varepsilon}$ of the following approximated problem

$$
\left\{\begin{array}{l}
u_{t}=-\varepsilon \nabla_{x}^{3} u_{x}+\nabla_{x}^{2} u_{x}+\rho \operatorname{Ric}\left(u_{x}, u_{x}\right) u_{x}, \quad x \in S^{1} ; \\
u(x, 0)=u_{0}(x) \in H^{k}\left(S^{1}, N\right) .
\end{array}\right.
$$

Then we have to derive the uniformly bound of $\left\|u_{\varepsilon x}\right\|_{H^{m}}^{2}$ which is independent of $\varepsilon$ such that we could obtain a limit $u(x, t)$ of the sequence $\left\{u_{\varepsilon}\right\}$ in suitable spaces as $\varepsilon$ goes to zero and it remains to show that the limit $u(x, t)$ is a solution of the Cauchy problem (1.4). However, because the different structure between the new flow and KdV geometric flow, we could not obtain the bounds of $\left\|u_{\varepsilon x}\right\|_{H^{m}}^{2}$ by calculating $\frac{d}{d t}\left\|u_{\varepsilon x}\right\|_{H^{m}}^{2}$ directly as we did in [27]. Precisely, if we differentiate $\left\|\nabla_{x}^{2} u_{\varepsilon x}\right\|_{L^{2}}^{2}$ with respect to $t$ and substitute (1.5), after integrating by parts, one would get terms as follows

$$
\int_{S^{1}} h\left(\nabla_{x}^{3} u_{\varepsilon x}, u_{\varepsilon x}\right) \operatorname{Ric}\left(\nabla_{x}^{2} u_{\varepsilon x}, u_{\varepsilon x}\right) d x
$$

which could not be bounded by $\left\|u_{\varepsilon x}\right\|_{H^{2}}^{2 s}$ for $s \in \mathbb{Z}^{+}$. Hence we have to try different ways to get those estimations. We find that, for a Riemannian manifold $N$ with parallel Ricci curvature, i.e. $\nabla$ Ric $\equiv 0$, if the Ricci curvature has a positive lower bound $\lambda>0$ (or a negative upper bound $-\lambda<0$ ), the Ricci tensor $\operatorname{Ric}(\cdot, \cdot)$ will have very nice properties which are similar with that of the metric $h(\cdot, \cdot)$. Instead estimating $\left\|u_{\varepsilon x}\right\|_{H^{k}}^{2}$, we could estimate

$$
I_{m}\left(u_{\varepsilon x}\right)=\sum_{i=0}^{m} \int_{S^{1}} \operatorname{Ric}\left(\nabla_{x}^{i} u_{\varepsilon x}, \nabla_{x}^{i} u_{\varepsilon x}\right) d x
$$

and derive the uniform bounds of $I_{m}$ on a time interval $(0, T)$ where both the bounds and $T$ are independent of $\varepsilon$. Then we would obtain the uniform bounds of $\left\|u_{x}\right\|_{H^{m}}^{2}$ since

$$
\left\|u_{x}\right\|_{H^{m}}^{2} \leq \frac{1}{\lambda} I_{m}\left(u_{\varepsilon x}\right)\left(\text { or }\left\|u_{x}\right\|_{H^{m}}^{2} \leq-\frac{1}{\lambda} I_{m}\left(u_{\varepsilon x}\right)\right) .
$$

These estimations will be derived in next section. In one word, the condition on the Ricci curvature of $N$ helps us obtain the uniform estimations about the approximated solution and its high order co-variant derivatives. Then, standard arguments are adopted to derive the local existence of (1.4).

Remark 1. It is well known that all irreducible symmetric spaces are Einstein. We should note that all the above results hold true on Einstein manifolds with a positive Einstein constant or a negative Einstein constant. However, if $N$ is Ricci flat, i.e. Ric $\equiv 0$, the new geometric flow then changed to

$$
\frac{\partial u}{\partial t}=\nabla_{x}^{2} u_{x}
$$

the method to discuss the existence in this work is ineffective. In this case, we could only obtain the local existence of the new flow via the same arguments as that in [27]. 
To prove the global existence we need to exploit some conservation laws and semi-conservation law. We define

$$
\begin{aligned}
E_{1}(u) \equiv & \int \operatorname{Ric}\left(u_{x}, u_{x}\right) d x \\
E_{2}(u) \equiv & \int \operatorname{Ric}\left(\nabla_{x} u_{x}, \nabla_{x} u_{x}\right) d x-\frac{\rho}{2} \int \operatorname{Ric}\left(u_{x}, u_{x}\right)^{2} d x \\
E_{3}(u) \equiv & 6 \int \operatorname{Ric}\left(\nabla_{x}^{2} u_{x}, \nabla_{x}^{2} u_{x}\right) d x-20 \rho \int \operatorname{Ric}\left(\nabla_{x} u_{x}, u_{x}\right)^{2} d x \\
& -10 \rho \int \operatorname{Ric}\left(\nabla_{x} u_{x}, \nabla_{x} u_{x}\right) \operatorname{Ric}\left(u_{x}, u_{x}\right) d x \\
& -4 \int \operatorname{Ric}\left(\nabla_{x} u_{x}, R\left(\nabla_{x} u_{x}, u_{x}\right) u_{x}\right) d x .
\end{aligned}
$$

If $N$ is a locally symmetric space, for the smooth solution $u$ to the Cauchy problem (1.4) we will establish the following in Sec.4:

$$
\frac{d}{d t} E_{1}(u)=0, \quad \frac{d}{d t} E_{2}(u)=0 .
$$

Moreover, if $N$ is a locally symmetric space on which the Ricci curvature has a positive lower bound $\lambda>0$ (or a negative upper bound $-\lambda<0$ ), then we have

$$
\frac{d}{d t} E_{3}(u) \leqslant C\left(N, \lambda, E_{1}\left(u_{0}\right), E_{2}\left(u_{0}\right)\right)\left(1+E_{3}\right) .
$$

We could make use of the above conservation laws with respect to $E_{1}(u)$ and $E_{2}(u)$ to derive a uniform a priori bound of $\left\|\nabla_{x} u_{x}\right\|_{L^{2}}$ independent of $T$. By virtue of (1.7), we will obtain the global existence results.

This paper is organized as follows: In Section 2 we employ the geometric energy method to establish the local existence of the new geometric flow. Since the conservation and semiconservation laws mentioned before are crucial for us to establish the global existence of the Cauchy problem of the geometric flow. We give a detailed calculation in Section 3. The global existence of the geometric flow on sepcial locally symmetric spaces is proved in Section 4 .

\section{Local Existence and Uniqueness}

In this section we establish the local existence and the uniqueness of solutions for the Cauchy problem of the new geometric flow (1.4) on a complete Riemannian manifold on which the Ricci curvature is parallel and has a positive lower bound $\lambda$, i.e. Ric $\geq \lambda>0$ (or a negative upper bound Ric $\leq-\lambda<0)$. We adopt the language that $\operatorname{Ric} \geq \lambda>0$ if all eigenvalues of $\operatorname{Ric}(X)$ are $\geq \lambda$. In $(0,2)$ language this means more precisely that $\operatorname{Ric}(X, X) \geq \lambda h(X, X)$ for all $X \in T N$. Moreover, if Ric $\geq \lambda>0$ on a complete Riemannian manifold $N$, then by Myers-Cheng's theorem we have $N$ is compact.

As in [27], to show the local existence of (1.4), we use the approximate method and discuss the following Cauchy problem:

$$
\left\{\begin{array}{l}
u_{t}=-\varepsilon \nabla_{x}^{3} u_{x}+\nabla_{x}^{2} u_{x}+\rho \operatorname{Ric}\left(u_{x}, u_{x}\right) u_{x}, \quad x \in S^{1} \\
u(x, 0)=u_{0}(x)
\end{array}\right.
$$


where $\varepsilon>0$ is a small positive constant.

We could imbed $N$ into a Euclidean space $\mathbb{R}^{n}$ for some large positive integer $n$. Then $N$ could be regarded as a submanifold of $\mathbb{R}^{n}$ and $u: S^{1} \times \mathbb{R} \rightarrow N \subset \mathbb{R}^{n}$ could be represented as $u=\left(u^{1}, \cdots, u^{n}\right)$ with $u^{i}$ being globally defined functions on $S^{1}$ so that the Sobolev-norms of $u$ make sense. We have

$$
\|u\|_{W^{m, 2}}^{2}=\sum_{i=0}^{m}\left\|D^{i} u\right\|_{L^{2}}^{2}
$$

where $D$ denotes the covariant derivative for functions on $S^{1}$. The equation (2.1) then becomes a fourth order parabolic system in $\mathbb{R}^{n}$. In the appendix of [27], we have shown that the parabolic equation admits a local solution $u_{\varepsilon} \in C\left(\left[0, T_{\varepsilon}\right), W^{k, 2}\left(S^{1}, N\right)\right)$ if the initial value map $u_{0} \in$ $W^{k, 2}\left(S^{1}, N\right)$ where $k \geq 3$.

Thus, in order to show the local existence of (1.4), we would find a uniform positive lower bound $T$ of $T_{\varepsilon}$ and uniform bounds for various norms of $u_{\varepsilon}(t)$ in suitable spaces for $t$ in the time interval $[0, T)$. Once we get these bounds it is easy to check that $u_{\varepsilon}$ subconverge to a strong solution of (1.4) as $\varepsilon \rightarrow 0$ via standard arguments.

Before proving the local existence, we shall introduce the properties about the Ricci curvature tensor and the Riemannina curvature $R$ on a Ricci parallel Riemannian manifold. We have

Proposition 2.1. Let $N$ be a Riemannian manifold with parallel Ricci curvature tensor, i.e. $\nabla R i c \equiv 0$. Then for $X, Y, Z, W \in \Gamma(T N)$, the Ricci curvature tensor and the Riemannian curvature tensor satisfy the following properties:

$$
\begin{aligned}
& \text { (1) } Z(\operatorname{Ric}(X, Y))=\operatorname{Ric}\left(\nabla_{Z} X, Y\right)+\operatorname{Ric}\left(X, \nabla_{Z} Y\right) \\
& \text { (2) } \operatorname{Ric}(X, R(Z, W) Y)=-\operatorname{Ric}(X, R(W, Z) Y)=\operatorname{Ric}(Z, R(X, Y) W) .
\end{aligned}
$$

These properties will be adopted frequently in the calculation throughout this paper. The process to show them is almost the same with proof of the symmetric and skew-symmetric properties of $R(X, Y, W, Z)$, we omit the details. Note that if $X \in \Gamma\left(u^{-1} T N\right)$ we have in local coordinates

and for $X=u_{x}$ we have

$$
\left(\nabla_{x} X\right)^{\alpha}=\frac{\partial X^{\alpha}}{\partial x}+\Gamma_{\beta \gamma}^{\alpha}(u) \frac{\partial u^{\beta}}{\partial x} X^{\gamma}
$$

$$
\left(\nabla_{t} u_{x}\right)^{\alpha}=\frac{\partial^{2} u^{\alpha}}{\partial t \partial x}+\Gamma_{\beta \gamma}^{\alpha}(u) \frac{\partial u^{\beta}}{\partial t} \frac{\partial u^{\gamma}}{\partial x} .
$$

It is easy to see that $\nabla_{t} u_{x}=\nabla_{x} u_{t}$.

Now we start the proof of the local existence of (1.4). Here we mainly discuss the case that Ric $\leq-\lambda<0$ on $N$ here. For the case Ric $\geq \lambda>0$, we could get the same results via easier arguments.

To begin with, let $u=u_{\varepsilon}$ be a solution of (2.1). We have the following results:

Lemma 2.2. (i) Assume that $N$ is a complete Riemannian manifold with parallel Ricci tensor, (i.e. $\nabla$ Ric $\equiv 0)$. If $N$ has negative upper bounds on the Ricci curvature (Ric $\leq-\lambda<0$ ) 
and uniform bounds on the curvature tensor $R$ and its covariant derivatives of any order (i.e., $\left.\left|\nabla^{l} R\right| \leq B_{l}, l=0,1,2, \cdots\right)$, and $u_{0} \in H^{k}\left(S^{1}, N\right)$ with an integer $k \geq 3$. Then there exists a constant $T=T\left(\left\|u_{0}\right\|_{H^{3}}\right)$, independent of $\varepsilon \in(0,1)$, such that if $u \in C\left(\left[0, T_{\varepsilon}\right), H^{k}\left(S^{1}, N\right)\right)$ is a solution of (2.1) with $\varepsilon \in(0,1)$, then $T\left(\left\|u_{0}\right\|_{H^{3}}\right) \leq T_{\varepsilon}$ and $\|u(t)\|_{H^{m+1}} \leq C\left(\left\|u_{0}\right\|_{H^{m+1}}\right)$ for any integer $2 \leq m \leq k-1$.

(ii) Assume that $N$ is a complete Riemannian manifold with parallel Ricci tensor and $N$ has negative upper bounds on the Ricci curvature. Let $u_{0} \in H^{k}\left(S^{1}, N\right)$ with an integer $k \geq 5$. Then there exists a constant $T=T\left(\left\|u_{0}\right\|_{H^{5}}\right)>0$, independent of $\varepsilon \in(0,1)$, such that if $u \in C\left(\left[0, T_{\varepsilon}\right), H^{k}\left(S^{1}, N\right)\right)$ is a solution of (2.1) with $\varepsilon \in(0,1)$, then $T\left(\left\|u_{0}\right\|_{H^{5}}\right) \leq T_{\varepsilon}$ and $\|u(t)\|_{H^{m+1}} \leq C\left(\left\|u_{0}\right\|_{H^{m+1}}\right)$ for any integer $2 \leq m \leq k-1$.

Proof. First fix a $k \geq 3$ and let $m$ be any integer with $2 \leq m \leq k-1$. We may assume that $u_{0}$ is $C^{\infty}$ smooth. Otherwise, we always choose a sequence of smooth functions $\left\{u_{0}^{i}\right\}$ such that $u_{0}^{i} \rightarrow u_{0}$ with respect to the norms $\|\cdot\|_{H^{k}}$ where $k \geq 3$.

As $N$ may not be compact we let, we let $\Omega \triangleq\left\{p \in N: \operatorname{dist}_{N}\left(p, u_{0}\left(S^{1}\right)\right)<1\right\}$, which is an open subset of $N$ with compact closure $\bar{\Omega}$. Let

$$
T^{\prime}=\sup \left\{t>0: u\left(S^{1}, t\right) \subset \Omega\right\} .
$$

Now we prove that if $k=3$, for all $t \in\left[0, T_{\varepsilon}\right]$

$$
\frac{d}{d t}\left(-\frac{1}{\lambda} \sum_{s=0}^{2} \int \operatorname{Ric}\left(\nabla_{x}^{s} u_{x}, \nabla_{x}^{s} u_{x}\right) d x\right) \leq C(\Omega, \lambda) \sum_{l=2}^{4}\left(-\frac{1}{\lambda} \sum_{s=0}^{2} \int \operatorname{Ric}\left(\nabla_{x}^{s} u_{x}, \nabla_{x}^{s} u_{x}\right) d x\right)^{l} .
$$

To see this, we first differentiate $\int \operatorname{Ric}\left(u_{x}, u_{x}\right) d x$ with respect to $t$ and we have:

$$
\begin{aligned}
& \frac{d}{d t} \int \operatorname{Ric}\left(u_{x}, u_{x}\right) d x \\
= & 2 \int \operatorname{Ric}\left(\nabla_{t} u_{x}, u_{x}\right) d x=2 \int \operatorname{Ric}\left(\nabla_{x} u_{t}, u_{x}\right) d x .
\end{aligned}
$$

Integrations by parts and substituting (2.1) yields

$$
\begin{aligned}
& \frac{d}{d t} \int \operatorname{Ric}\left(u_{x}, u_{x}\right) d x=-2 \int \operatorname{Ric}\left(u_{t}, \nabla_{x} u_{x}\right) d x \\
= & 2 \varepsilon \int \operatorname{Ric}\left(\nabla_{x}^{3} u_{x}, \nabla_{x} u_{x}\right) d x-2 \int \operatorname{Ric}\left(\nabla_{x}^{2} u_{x}, \nabla_{x} u_{x}\right) d x \\
& -2 \rho \int \operatorname{Ric}\left(\operatorname{Ric}\left(u_{x}, u_{x}\right) u_{x}, \nabla_{x} u_{x}\right) d x \\
= & -2 \varepsilon \int \operatorname{Ric}\left(\nabla_{x}^{2} u_{x}, \nabla_{x}^{2} u_{x}\right) d x-2 \int \nabla_{x}\left(\operatorname{Ric}\left(\nabla_{x} u_{x}, \nabla_{x} u_{x}\right)\right) d x \\
& -\frac{\rho}{2} \int \nabla_{x}\left(\left|\operatorname{Ric}\left(u_{x}, u_{x}\right)\right|^{2}\right) d x \\
= & -2 \varepsilon \int \operatorname{Ric}\left(\nabla_{x}^{2} u_{x}, \nabla_{x}^{2} u_{x}\right) d x
\end{aligned}
$$


Hence by the condition $R i c \leq-\lambda<0$ we have

$$
\begin{aligned}
\frac{d}{d t}\left(-\frac{1}{\lambda} \int \operatorname{Ric}\left(u_{x}, u_{x}\right) d x\right) & =\frac{2 \varepsilon}{\lambda} \int \operatorname{Ric}\left(\nabla_{x}^{2} u_{x}, \nabla_{x}^{2} u_{x}\right) d x \\
& \leq-2 \varepsilon \int\left|\nabla_{x}^{2} u_{x}\right|^{2} d x \leq 0
\end{aligned}
$$

Considering $\int \operatorname{Ric}\left(\nabla_{x} u_{x}, \nabla_{x} u_{x}\right) d x$ we have:

$$
\begin{aligned}
& \frac{d}{d t} \int \operatorname{Ric}\left(\nabla_{x} u_{x}, \nabla_{x} u_{x}\right) d x=2 \int \operatorname{Ric}\left(\nabla_{t} \nabla_{x} u_{x}, \nabla_{x} u_{x}\right) d x \\
= & 2 \int \operatorname{Ric}\left(\nabla_{x} \nabla_{t} u_{x}, \nabla_{x} u_{x}\right) d x+2 \int \operatorname{Ric}\left(R\left(u_{t}, u_{x}\right) u_{x}, \nabla_{x} u_{x}\right) d x \\
= & 2 \int \operatorname{Ric}\left(\nabla_{x}^{2} u_{t}, \nabla_{x} u_{x}\right) d x+2 \int \operatorname{Ric}\left(u_{t}, R\left(\nabla_{x} u_{x}, u_{x}\right) u_{x}\right) d x \\
= & 2 \int \operatorname{Ric}\left(u_{t}, \nabla_{x}^{3} u_{x}\right) d x+2 \int \operatorname{Ric}\left(u_{t}, R\left(\nabla_{x} u_{x}, u_{x}\right) u_{x}\right) d x .
\end{aligned}
$$

Thus, substituting (2.1) into above we have

$$
\begin{aligned}
& \frac{d}{d t} \int \operatorname{Ric}\left(\nabla_{x} u_{x}, \nabla_{x} u_{x}\right) d x \\
= & -2 \varepsilon \int \operatorname{Ric}\left(\nabla_{x}^{3} u_{x}, \nabla_{x}^{3} u_{x}\right) d x+2 \int \operatorname{Ric}\left(\nabla_{x}^{2} u_{x}, \nabla_{x}^{3} u_{x}\right) d x \\
& +2 \rho \int \operatorname{Ric}\left(u_{x}, u_{x}\right) \operatorname{Ric}\left(u_{x}, \nabla_{x}^{3} u_{x}\right) d x \\
& -2 \varepsilon \int \operatorname{Ric}\left(\nabla_{x}^{3} u_{x}, R\left(\nabla_{x} u_{x}, u_{x}\right) u_{x}\right) d x+2 \int \operatorname{Ric}\left(\nabla_{x}^{2} u_{x}, R\left(\nabla_{x} u_{x}, u_{x}\right) u_{x}\right) d x \\
& +2 \rho \int \operatorname{Ric}\left(u_{x}, u_{x}\right) \operatorname{Ric}\left(u_{x}, R\left(\nabla_{x} u_{x}, u_{x}\right) u_{x}\right) d x .
\end{aligned}
$$

It is easy to see that the second term and the last term on the right hand side vanish since

$$
2 \operatorname{Ric}\left(\nabla_{x}^{2} u_{x}, \nabla_{x}^{3} u_{x}\right)=\nabla_{x}\left(\operatorname{Ric}\left(\nabla_{x}^{2} u_{x}, \nabla_{x}^{2} u_{x}\right)\right)
$$

and

$$
\operatorname{Ric}\left(u_{x}, R\left(\nabla_{x} u_{x}, u_{x}\right) u_{x}\right) \equiv 0 .
$$

Moreover, for the fifth term on the right, we have

$$
\begin{aligned}
& 2 \int \operatorname{Ric}\left(\nabla_{x}^{2} u_{x}, R\left(\nabla_{x} u_{x}, u_{x}\right) u_{x}\right) d x \\
= & -2 \int \operatorname{Ric}\left(\nabla_{x} u_{x},\left(\nabla_{x} R\right)\left(\nabla_{x} u_{x}, u_{x}\right) u_{x}\right) d x-2 \int \operatorname{Ric}\left(\nabla_{x} u_{x}, R\left(\nabla_{x}^{2} u_{x}, u_{x}\right) u_{x}\right) d x,
\end{aligned}
$$

which implies that

$$
2 \int \operatorname{Ric}\left(\nabla_{x}^{2} u_{x}, R\left(\nabla_{x} u_{x}, u_{x}\right) u_{x}\right) d x=-\int \operatorname{Ric}\left(\nabla_{x} u_{x},\left(\nabla_{x} R\right)\left(\nabla_{x} u_{x}, u_{x}\right) u_{x}\right) d x .
$$


For the left terms of (2.5), after integration by parts we get

$$
\begin{aligned}
& 2 \rho \int \operatorname{Ric}\left(u_{x}, u_{x}\right) \operatorname{Ric}\left(u_{x}, \nabla_{x}^{3} u_{x}\right) d x \\
= & -2 \rho \int \operatorname{Ric}\left(u_{x}, u_{x}\right) \operatorname{Ric}\left(\nabla_{x} u_{x}, \nabla_{x}^{2} u_{x}\right) d x-4 \rho \int \operatorname{Ric}\left(\nabla_{x} u_{x}, u_{x}\right) \operatorname{Ric}\left(u_{x}, \nabla_{x}^{2} u_{x}\right) d x \\
= & 6 \rho \int \operatorname{Ric}\left(\nabla_{x} u_{x}, \nabla_{x} u_{x}\right) \operatorname{Ric}\left(\nabla_{x} u_{x}, u_{x}\right) d x
\end{aligned}
$$

and

$$
\begin{aligned}
& -2 \varepsilon \int \operatorname{Ric}\left(\nabla_{x}^{3} u_{x}, R\left(\nabla_{x} u_{x}, u_{x}\right) u_{x}\right) d x \\
= & 2 \varepsilon \int \operatorname{Ric}\left(\nabla_{x}^{2} u_{x},\left(\nabla_{x} R\right)\left(\nabla_{x} u_{x}, u_{x}\right) u_{x}\right) d x+2 \varepsilon \int \operatorname{Ric}\left(\nabla_{x}^{2} u_{x}, R\left(\nabla_{x}^{2} u_{x}, u_{x}\right) u_{x}\right) d x \\
& +2 \varepsilon \int \operatorname{Ric}\left(\nabla_{x}^{2} u_{x}, R\left(\nabla_{x} u_{x}, u_{x}\right) \nabla_{x} u_{x}\right) d x .
\end{aligned}
$$

Hence we obtain that

$$
\begin{aligned}
& \frac{d}{d t}\left(-\frac{1}{\lambda} \int \operatorname{Ric}\left(\nabla_{x} u_{x}, \nabla_{x} u_{x}\right) d x\right)-\frac{2 \varepsilon}{\lambda} \int \operatorname{Ric}\left(\nabla_{x}^{3} u_{x}, \nabla_{x}^{3} u_{x}\right) d x \\
= & -\frac{1}{\lambda}\left(2 \varepsilon \int \operatorname{Ric}\left(\nabla_{x}^{2} u_{x},\left(\nabla_{x} R\right)\left(\nabla_{x} u_{x}, u_{x}\right) u_{x}\right) d x+2 \varepsilon \int \operatorname{Ric}\left(\nabla_{x}^{2} u_{x}, R\left(\nabla_{x}^{2} u_{x}, u_{x}\right) u_{x}\right) d x\right. \\
& +2 \varepsilon \int \operatorname{Ric}\left(\nabla_{x}^{2} u_{x}, R\left(\nabla_{x} u_{x}, u_{x}\right) \nabla_{x} u_{x}\right) d x-\int \operatorname{Ric}\left(\nabla_{x} u_{x},\left(\nabla_{x} R\right)\left(\nabla_{x} u_{x}, u_{x}\right) u_{x}\right) d x \\
& \left.+6 \rho \int \operatorname{Ric}\left(\nabla_{x} u_{x}, \nabla_{x} u_{x}\right) \operatorname{Ric}\left(\nabla_{x} u_{x}, u_{x}\right) d x\right) \\
\leq & C(\Omega, \rho, \lambda)\left(\int\left|\nabla_{x}^{2} u_{x}\right|\left|\nabla_{x} u_{x}\right|\left|u_{x}\right|^{3} d x+\int\left|\nabla_{x}^{2} u_{x}\right|^{2}\left|u_{x}\right|^{2} d x+\int\left|\nabla_{x}^{2} u_{x}\right|\left|\nabla_{x} u_{x}\right|^{2}\left|u_{x}\right| d x\right. \\
& \left.+\int\left|\nabla_{x} u_{x}\right|^{2}\left|u_{x}\right|^{3} d x+\int\left|\nabla_{x} u_{x}\right|^{3}\left|u_{x}\right| d x\right) .
\end{aligned}
$$

Utilizing Hölder inequality and the following interpolation inequalities

$$
\begin{aligned}
\left\|u_{x}\right\|_{L^{\infty}} & \leq C(\Omega)\left(\left\|\nabla_{x} u_{x}\right\|_{L^{2}}^{2}+\left\|u_{x}\right\|_{L^{2}}^{2}\right)^{\frac{1}{4}}\left\|u_{x}\right\|_{L^{2}}^{\frac{1}{2}} ; \\
\left\|\nabla_{x} u_{x}\right\|_{L^{\infty}} & \leq C(\Omega)\left(\left\|\nabla_{x}^{2} u_{x}\right\|_{L^{2}}^{2}+\left\|\nabla_{x} u_{x}\right\|_{L^{2}}^{2}\right)^{\frac{1}{4}}\left\|\nabla_{x} u_{x}\right\|_{L^{2}}^{\frac{1}{2}},
\end{aligned}
$$

we obtain that

$$
\frac{d}{d t}\left(-\frac{1}{\lambda} \int \operatorname{Ric}\left(\nabla_{x} u_{x}, \nabla_{x} u_{x}\right) d x\right)-\frac{2 \varepsilon}{\lambda} \int \operatorname{Ric}\left(\nabla_{x}^{3} u_{x}, \nabla_{x}^{3} u_{x}\right) d x \leq C(\Omega, \rho, \lambda)\left\|u_{x}\right\|_{H^{2}}^{4} .
$$

Now to show (2.3), we need compute $\frac{d}{d t} \int \operatorname{Ric}\left(\nabla_{x}^{2} u_{x}, \nabla_{x}^{2} u_{x}\right) d x$ and we have

$$
\frac{d}{d t} \int \operatorname{Ric}\left(\nabla_{x}^{2} u_{x}, \nabla_{x}^{2} u_{x}\right) d x=2 \int \operatorname{Ric}\left(\nabla_{t} \nabla_{x}^{2} u_{x}, \nabla_{x}^{2} u_{x}\right) d x
$$




$$
\begin{aligned}
= & 2 \int \operatorname{Ric}\left(\nabla_{x} \nabla_{t} \nabla_{x} u_{x}, \nabla_{x}^{2} u_{x}\right) d x+2 \int \operatorname{Ric}\left(R\left(u_{t}, u_{x}\right) \nabla_{x} u_{x}, \nabla_{x}^{2} u_{x}\right) d x \\
= & -2 \int \operatorname{Ric}\left(\nabla_{x}^{2} u_{t}, \nabla_{x}^{3} u_{x}\right) d x-2 \int \operatorname{Ric}\left(\nabla_{x}^{3} u_{x}, R\left(u_{t}, u_{x}\right) u_{x}\right) d x \\
& +2 \int \operatorname{Ric}\left(\nabla_{x}^{2} u_{x}, R\left(u_{t}, u_{x}\right) \nabla_{x} u_{x}\right) d x \\
= & -2 \int \operatorname{Ric}\left(u_{t}, \nabla_{x}^{5} u_{x}\right) d x-2 \int \operatorname{Ric}\left(u_{t}, R\left(\nabla_{x}^{3} u_{x}, u_{x}\right) u_{x}\right) d x \\
& +2 \int \operatorname{Ric}\left(u_{t}, R\left(\nabla_{x}^{2} u_{x}, \nabla_{x} u_{x}\right) u_{x}\right) d x .
\end{aligned}
$$

Substituting (2.1) into (2.9) and noting that

$$
\begin{gathered}
\int \operatorname{Ric}\left(\nabla_{x}^{3} u_{x}, \nabla_{x}^{5} u_{x}\right) d x=-\int \operatorname{Ric}\left(\nabla_{x}^{4} u_{x}, \nabla_{x}^{4} u_{x}\right) ; \int \operatorname{Ric}\left(\nabla_{x}^{2} u_{x}, \nabla_{x}^{5} u_{x}\right) d x=0 \\
\int \operatorname{Ric}\left(u_{x}, R\left(\nabla_{x}^{3} u_{x}, u_{x}\right) u_{x}\right) d x=\int \operatorname{Ric}\left(u_{x}, R\left(\nabla_{x}^{2} u_{x}, \nabla_{x} u_{x}\right) u_{x}\right) \equiv 0
\end{gathered}
$$

we have

$$
\begin{aligned}
& \frac{d}{d t}\left(-\frac{1}{\lambda} \int \operatorname{Ric}\left(\nabla_{x}^{2} u_{x}, \nabla_{x}^{2} u_{x}\right) d x\right)-\frac{2 \varepsilon}{\lambda} \int \operatorname{Ric}\left(\nabla_{x}^{4} u_{x}, \nabla_{x}^{4} u_{x}\right) d x \\
= & -\frac{1}{\lambda}\left(2 \varepsilon \int \operatorname{Ric}\left(\nabla_{x}^{3} u_{x}, R\left(\nabla_{x}^{3} u_{x}, u_{x}\right) u_{x}\right) d x-2 \varepsilon \int \operatorname{Ric}\left(\nabla_{x}^{3} u_{x}, R\left(\nabla_{x}^{2} u_{x}, \nabla_{x} u_{x}\right) u_{x}\right) d x\right. \\
& -2 \rho \int \operatorname{Ric}\left(\operatorname{Ric}\left(u_{x}, u_{x}\right) u_{x}, \nabla_{x}^{5} u_{x}\right) d x-2 \int \operatorname{Ric}\left(\nabla_{x}^{2} u_{x}, R\left(\nabla_{x}^{3} u_{x}, u_{x}\right) u_{x}\right) d x \\
& \left.+2 \int \operatorname{Ric}\left(\nabla_{x}^{2} u_{x}, R\left(\nabla_{x}^{2} u_{x}, \nabla_{x} u_{x}\right) u_{x}\right) d x .\right)
\end{aligned}
$$

For the first two terms of (2.10) on the right, integrating by parts yields

$$
\begin{aligned}
& 2 \varepsilon \int \operatorname{Ric}\left(\nabla_{x}^{3} u_{x}, R\left(\nabla_{x}^{3} u_{x}, u_{x}\right) u_{x}\right) d x-2 \varepsilon \int \operatorname{Ric}\left(\nabla_{x}^{3} u_{x}, R\left(\nabla_{x}^{2} u_{x}, \nabla_{x} u_{x}\right) u_{x}\right) d x \\
= & -2 \varepsilon \int \operatorname{Ric}\left(\nabla_{x}^{3} u_{x},\left(\nabla_{x} R\right)\left(\nabla_{x}^{2} u_{x}, u_{x}\right) u_{x}\right) d x-2 \varepsilon \int \operatorname{Ric}\left(\nabla_{x}^{4} u_{x}, R\left(\nabla_{x}^{2} u_{x}, u_{x}\right) u_{x}\right) d x \\
& -2 \varepsilon \int \operatorname{Ric}\left(\nabla_{x}^{3} u_{x}, R\left(\nabla_{x}^{2} u_{x}, u_{x}\right) \nabla_{x} u_{x}\right) d x \\
& -4 \varepsilon \int \operatorname{Ric}\left(\nabla_{x}^{3} u_{x}, R\left(\nabla_{x}^{2} u_{x}, \nabla_{x} u_{x}\right) u_{x}\right) d x .
\end{aligned}
$$

Hence for any $\delta>0$,

$$
\begin{aligned}
& -\frac{1}{\lambda}\left(2 \varepsilon \int \operatorname{Ric}\left(\nabla_{x}^{3} u_{x}, R\left(\nabla_{x}^{3} u_{x}, u_{x}\right) u_{x}\right) d x-2 \varepsilon \int \operatorname{Ric}\left(\nabla_{x}^{3} u_{x}, R\left(\nabla_{x}^{2} u_{x}, \nabla_{x} u_{x}\right) u_{x}\right) d x\right) \\
\leq & \frac{\varepsilon \delta}{\lambda} \int\left|\nabla_{x}^{4} u_{x}\right|^{2} d x+\frac{4 \varepsilon \delta}{\lambda} \int\left|\nabla_{x}^{3} u_{x}\right|^{2} d x \\
& +\frac{\varepsilon C(\Omega)}{2 \delta \lambda}\left\{\int\left|\left(\nabla_{x} R\right)\left(\nabla_{x}^{2} u_{x}, u_{x}\right) u_{x}\right|^{2} d x+\int\left|R\left(\nabla_{x}^{2} u_{x}, u_{x}\right) u_{x}\right|^{2} d x\right.
\end{aligned}
$$




$$
\begin{aligned}
& \left.+\int\left|R\left(\nabla_{x}^{2} u_{x}, u_{x}\right) \nabla_{x} u_{x}\right|^{2} d x+2 \int\left|R\left(\nabla_{x}^{2} u_{x}, \nabla_{x} u_{x}\right) u_{x}\right|^{2} d x\right\} \\
\leq & \frac{\varepsilon \delta}{\lambda} \int\left|\nabla_{x}^{4} u_{x}\right|^{2} d x+\frac{4 \varepsilon \delta}{\lambda} \int\left|\nabla_{x}^{3} u_{x}\right|^{2} d x \\
& +\frac{C(\Omega)}{2 \delta \lambda} \int\left|\nabla_{x}^{2} u_{x}\right|^{2}\left(\left|u_{x}\right|^{6}+\left|u_{x}\right|^{4}+\left|\nabla_{x} u_{x}\right|^{2}\left|u_{x}\right|^{2}\right) d x \\
\leq & \frac{\varepsilon \delta}{\lambda} \int\left|\nabla_{x}^{4} u_{x}\right|^{2} d x+\frac{4 \varepsilon \delta}{\lambda} \int\left|\nabla_{x}^{3} u_{x}\right|^{2} d x+\frac{C(\Omega)}{2 \delta \lambda}\left(|| u_{x}\left\|_{H^{2}}^{4}+\right\| u_{x}\left\|_{H^{2}}^{6}+\right\| u_{x} \|_{H^{2}}^{8}\right) \\
\leq & -\frac{\varepsilon \delta}{\lambda^{2}} \int \operatorname{Ric}\left(\nabla_{x}^{4} u_{x}, \nabla_{x}^{4} u_{x}\right) d x-\frac{4 \varepsilon \delta}{\lambda^{2}} \int \operatorname{Ric}\left(\nabla_{x}^{3} u_{x}, \nabla_{x}^{3} u_{x}\right) d x \\
& +\frac{C(\Omega)}{2 \delta \lambda}\left(\left\|u_{x}\right\|_{H^{2}}^{4}+\left\|u_{x}\right\|_{H^{2}}^{6}+|| u_{x} \|_{H^{2}}^{8}\right) .
\end{aligned}
$$

For the third term of (2.10), integrating by parts yields

$$
\begin{aligned}
& -\int \operatorname{Ric}\left(\operatorname{Ric}\left(u_{x}, u_{x}\right) u_{x}, \nabla_{x}^{5} u_{x}\right) d x \\
= & \int \operatorname{Ric}\left(\nabla_{x}^{4} u_{x}, \nabla_{x} u_{x}\right) \operatorname{Ric}\left(u_{x}, u_{x}\right) d x+2 \int \operatorname{Ric}\left(\nabla_{x}^{4} u_{x}, u_{x}\right) \operatorname{Ric}\left(\nabla_{x} u_{x}, u_{x}\right) d x \\
= & -\int \operatorname{Ric}\left(\nabla_{x}^{3} u_{x}, \nabla_{x}^{2} u_{x}\right) \operatorname{Ric}\left(u_{x}, u_{x}\right) d x-4 \int \operatorname{Ric}\left(\nabla_{x}^{3} u_{x}, \nabla_{x} u_{x}\right) \operatorname{Ric}\left(\nabla_{x} u_{x}, u_{x}\right) d x \\
& -2 \int \operatorname{Ric}\left(\nabla_{x}^{3} u_{x}, u_{x}\right) \operatorname{Ric}\left(\nabla_{x}^{2} u_{x}, u_{x}\right) d x-2 \int \operatorname{Ric}\left(\nabla_{x}^{3} u_{x}, u_{x}\right) \operatorname{Ric}\left(\nabla_{x} u_{x}, \nabla_{x} u_{x}\right) d x \\
= & 5 \int \operatorname{Ric}\left(\nabla_{x}^{2} u_{x}, \nabla_{x}^{2} u_{x}\right) \operatorname{Ric}\left(\nabla_{x} u_{x}, u_{x}\right) d x+10 \int \operatorname{Ric}\left(\nabla_{x}^{2} u_{x}, \nabla_{x} u_{x}\right) \operatorname{Ric}\left(\nabla_{x}^{2} u_{x}, u_{x}\right) d x \\
\leq & C(\Omega) \int\left|\nabla_{x}^{2} u_{x}\right|^{2}\left|\nabla_{x} u_{x}\right|\left|u_{x}\right| d x \leq C(\Omega)|| \nabla_{x} u_{x}\left\|_{L^{\infty}}|| u_{x}\right\|_{L^{\infty}} \int\left|\nabla_{x}^{2} u_{x}\right|^{2} d x
\end{aligned}
$$

Thus by (2.7) we have

$$
\frac{2 \rho}{\lambda} \int \operatorname{Ric}\left(\operatorname{Ric}\left(u_{x}, u_{x}\right) u_{x}, \nabla_{x}^{5} u_{x}\right) d x \leq C(\Omega, \rho, \lambda)\left\|u_{x}\right\|_{H^{2}}^{4} .
$$

It is easy to check that the other two terms of (2.10) are also bounded by by $C(\Omega)\left\|u_{x}\right\|_{H^{2}}^{4}$ via the similar argument, we omit the detail. This together with Ineq.(2.4), (2.8) and (2.10)-(2.14) yields

$$
\begin{aligned}
0< & \frac{d}{d t} \int\left(-\frac{1}{\lambda}\right)\left\{\operatorname{Ric}\left(\nabla_{x}^{2} u_{x}, \nabla_{x}^{2} u_{x}\right)+\operatorname{Ric}\left(\nabla_{x} u_{x}, \nabla_{x} u_{x}\right)+\operatorname{Ric}\left(u_{x}, u_{x}\right)\right\} d x \\
& -\left(\frac{2 \varepsilon}{\lambda}-\frac{\varepsilon \delta}{\lambda^{2}}\right) \int \operatorname{Ric}\left(\nabla_{x}^{4} u_{x}, \nabla_{x}^{4} u_{x}\right) d x-\left(\frac{2 \varepsilon}{\lambda}-\frac{4 \varepsilon \delta}{\lambda^{2}}\right) \int \operatorname{Ric}\left(\nabla_{x}^{3} u_{x}, \nabla_{x}^{3} u_{x}\right) d x \\
\leq & C(\Omega, \rho, \lambda)\left(\frac{1}{2 \delta}+1\right)\left(\left\|u_{x}\right\|_{H^{2}}^{4}+\left\|u_{x}\right\|_{H^{2}}^{6}+\left\|u_{x}\right\|_{H^{2}}^{8}\right) .
\end{aligned}
$$

Thus, let $\delta=\frac{\lambda}{8}$, we have

$$
0<\frac{d}{d t}\left(-\frac{1}{\lambda} \sum_{s=0}^{2} \int \operatorname{Ric}\left(\nabla_{x}^{s} u_{x}, \nabla_{x}^{s} u_{x}\right) d x\right) \leq C(\Omega, \rho, \lambda) \sum_{l=2}^{4}\left\|u_{x}\right\|_{H^{2}}^{2 l} .
$$


Furthermore, by the assumption that the Ricci curvature on $N$ has a negative upper bound $-\lambda$, we have

$$
\left\|u_{x}\right\|_{H^{2}}^{2}=\sum_{s=0}^{2} \int\left|\nabla_{x}^{s} u_{x}\right|^{2} d x \leq-\frac{1}{\lambda} \sum_{s=0}^{2} \int \operatorname{Ric}\left(\nabla_{x}^{s} u_{x}, \nabla_{x}^{s} u_{x}\right) d x .
$$

Hence from (2.16) we could obtain that

$$
\frac{d}{d t}\left(-\frac{1}{\lambda} \sum_{s=0}^{2} \int \operatorname{Ric}\left(\nabla_{x}^{s} u_{x}, \nabla_{x}^{s} u_{x}\right) d x\right) \leq C(\Omega, \rho, \lambda) \sum_{l=2}^{4}\left(-\frac{1}{\lambda} \sum_{s=0}^{2} \int \operatorname{Ric}\left(\nabla_{x}^{s} u_{x}, \nabla_{x}^{s} u_{x}\right) d x\right)^{l}
$$

If $k \geq 4$, then for $3 \leq m \leq k-1$, by the similar argument, we could get

$$
\frac{d}{d t}\left(-\frac{1}{\lambda} \sum_{s=0}^{m} \int \operatorname{Ric}\left(\nabla_{x}^{s} u_{x}, \nabla_{x}^{s} u_{x}\right) d x\right) \leq C\left(\Omega, \rho, \lambda, Q_{m-1}\right)\left(-\frac{1}{\lambda}\right) \sum_{s=0}^{m} \int \operatorname{Ric}\left(\nabla_{x}^{s} u_{x}, \nabla_{x}^{s} u_{x}\right) d x
$$

where

$$
Q_{m-1}(u)=-\frac{1}{\lambda} \sum_{i=0}^{m-1} \int \operatorname{Ric}\left(\nabla_{x}^{i} u_{x}, \nabla_{x}^{i} u_{x}\right) d x \geq 0,
$$

$C\left(\Omega, \rho, \lambda, Q_{m-1}\right)$ only depends on $Q_{m-1}, \rho, \lambda$, the bounds on the Ricci curvature and the bounds on the curvature $R$ and its covariant derivatives $\nabla^{l} R$ with $l \leq m$ on $\Omega \subset N$. We omit the details of the proof. We should note that by the definition of $Q_{m-1}(u)$ we have

$$
Q_{m-1}(u) \leq C(\Omega, \lambda) \sum_{i=0}^{m-1} \int\left|\nabla_{x}^{i} u_{x}\right|^{2} d x=C(\Omega)\left\|u_{x}\right\|_{H^{m-1}}^{2}
$$

Thus, if we let

$$
f(t)=-\frac{1}{\lambda} \sum_{s=0}^{2} \int \operatorname{Ric}\left(\nabla_{x}^{s} u_{x}, \nabla_{x}^{s} u_{x}\right) d x+1
$$

then we have

$$
\frac{d f}{d t} \leq C(\Omega) f^{4}, \quad f(0)=-\frac{1}{\lambda} \sum_{s=0}^{2} \int \operatorname{Ric}\left(\nabla_{x}^{s} u_{0 x}, \nabla_{x}^{s} u_{0 x}\right) d x+1 .
$$

It follows from (2.19) that there exists constants $T_{0}>0$ and $C_{0}>0$ such that

$$
\left\|u_{x}\right\|_{H^{2}}^{2} \leq-\frac{1}{\lambda} \sum_{s=0}^{2} \int \operatorname{Ric}\left(\nabla_{x}^{s} u_{x}, \nabla_{x}^{s} u_{x}\right) d x \leq C_{0}, \quad t \in\left[0, \min \left(T_{0}, T^{\prime}\right)\right] .
$$

Now let $T=\min \left(T_{0}, T^{\prime}\right)$. If $m=3$, by the Gronwall inequality, we can obtain from (2.18):

$$
\left\|u_{x}\right\|_{H^{3}}^{2} \leq-\frac{1}{\lambda} \sum_{s=0}^{3} \int \operatorname{Ric}\left(\nabla_{x}^{s} u_{x}, \nabla_{x}^{s} u_{x}\right) d x \leq C_{1}\left(\Omega, \lambda, T, Q_{3}\left(u_{0}\right)\right), \quad \text { for all } \quad t \in[0, T] .
$$


Then by induction we have that there exists a constant $C_{m-2}\left(\Omega, \lambda, Q_{m}\left(u_{0}\right)\right)>0$, such that for any $3 \leq m \leq k-1$

$$
\operatorname{ess} \sup _{t \in[0, T]}\left\|u_{x}\right\|_{H^{m}}^{2} \leq C_{m-2}\left(\Omega, \lambda, Q_{m}\left(u_{0}\right)\right) \leq C_{m-2}\left(\Omega, \lambda,\left\|u_{0 x}\right\|_{H^{m}}\right) .
$$

If $N$ is of uniform bounds on the curvature tensor and its derivatives $\nabla^{l} R$ with $l \leq m$, it is easy to see from the above arguments that $T=T_{0}$ since the coefficients of the above differential inequalities depend only on the bounds on Ricci curvature, the Riemann curvature tensor $R$ and its covariant derivatives $\nabla^{l} R$ of order $l \leq m$ on $N$. That is $T=T\left(N, Q_{3}\left(u_{0}\right), \lambda\right)$ depends only on $N, u_{0}$ and $\lambda$, not on $0<\varepsilon<1$.

Now we consider the case $N$ is a noncompact, complete Riemannian manifold with parallel Ricci tensor and the Ricci curvature has a negative upper bound $-\lambda<0$. Note that a positive lower bound of $T^{\prime}$ can also be derived from (2.20) when $k \geq 5$. Indeed, It is easy to see from the approximate equation (2.1) and the interpolation inequalities (see Theorem 2.1 in [8] for details) that (2.20) implies

$$
\operatorname{ess} \sup _{t \in[0, T]} \mid\left\|u_{t}\right\|_{L^{2}\left(S^{1}, T N\right)} \leq C\left(\Omega, \lambda, Q_{3}\left(u_{0}\right)\right) \leq C\left(\Omega, \lambda,\left\|u_{0 x}\right\|_{H^{3}}\right) .
$$

On the other hand, from the approximate equation of the geometric flow (2.1) we have

$$
\nabla_{x} u_{t}=-\varepsilon \nabla_{x}^{4} u_{x}+\nabla_{x}^{3} u_{x}+\rho \nabla_{x}\left(\operatorname{Ric}\left(u_{x}, u_{x}\right) u_{x}\right) .
$$

Hence, when $k \geq 5$ we infer from (2.20) and the interpolation inequality that

$$
\operatorname{ess} \sup _{t \in[0, T]}\left\|u_{t}\right\|_{H^{1}\left(S^{1}, T N\right)} \leq C\left(\Omega,\left\|u_{0 x}\right\|_{H^{4}}\right) .
$$

Moreover, for some $0<a<1$ the following interpolation inequality holds

$$
\left\|u_{t}(s)\right\|_{L^{\infty}} \leq C\left\|u_{t}(s)\right\|_{H^{1}}^{a}\left\|u_{t}(s)\right\|_{L^{2}}^{1-a} .
$$

This implies that, for some $\mathcal{M}>0$, there holds true

$$
\operatorname{ess} \sup _{t \in[0, T]}\left\|u_{t}\right\|_{L^{\infty}} \leq \mathcal{M} \text {. }
$$

Thus we have

$$
\sup _{x \in S^{1}} d_{N}\left(u(x, t), u_{0}(x)\right) \leq \mathcal{M} t, \quad \text { for } \quad t<T .
$$

If $T^{\prime}>T_{0}$ we get the lower bound, so we may assume that $T^{\prime} \leq T_{0}$. Then letting $t \rightarrow T^{\prime}$ in the above inequality we get $\mathcal{M} T^{\prime} \geq 1$. Therefore, if we set $T=\min \left\{\frac{1}{\mathcal{M}}, T_{0}\right\}$, then the desired estimates hold for $t \in[0, T]$.

It is easy to find that the solution to $(2.1)$ with $\varepsilon \in(0,1)$ must exist on the time interval $[0, T]$. Otherwise, we always extend the time interval of existence to cover $[0, T]$. Hence we always have $T_{\varepsilon} \geq T$ and then we complete the proof of this lemma.

Here we should point out that if the ricci curvature of a complete Riemannian manifold $N$ has a positive lower bound, i.e. Ric $\geq \lambda>0$, then by Myers's theorem $N$ must be compact. Hence in this case we have the following corollary via easier arguments than that in Lemma 2.2. 
Corollary 2.3. Assume that $N$ is a complete Riemannian manifold with parallel Ricci tensor, (i.e. $\nabla$ Ric $\equiv 0)$ ). If $N$ has positive lower bounds on the Ricci curvature (Ric $\geq \lambda>0$ ) and $u_{0} \in H^{k}\left(S^{1}, N\right)$ with an integer $k \geq 3$. Then there exists a constant $T=T\left(\left\|u_{0}\right\|_{H^{3}}\right)$, independent of $\varepsilon \in(0,1)$, such that if $u \in C\left(\left[0, T_{\varepsilon}\right), H^{k}\left(S^{1}, N\right)\right)$ is a solution of (2.1) with $\varepsilon \in(0,1)$, then $T\left(\left\|u_{0}\right\|_{H^{3}}\right) \leq T_{\varepsilon}$ and $\|u(t)\|_{H^{m+1}} \leq C\left(\left\|u_{0}\right\|_{H^{m+1}}\right)$ for any integer $2 \leq m \leq k-1$.

We omit the details of the proof since the process is similar and in this case $N$ is compact. Here we point out that instead of showing (2.3), it is suffices to show

$$
\frac{d}{d t}\left(\sum_{s=0}^{2} \int \operatorname{Ric}\left(\nabla_{x}^{s} u_{x}, \nabla_{x}^{s} u_{x}\right) d x\right) \leq C(\Omega, \lambda) \sum_{l=2}^{4}\left(\sum_{s=0}^{2} \int \operatorname{Ric}\left(\nabla_{x}^{s} u_{x}, \nabla_{x}^{s} u_{x}\right) d x\right)^{l} .
$$

Following Lemma2.2, we could obtain the following local existence results of the Cauchy problem (1.4).

Lemma 2.4. Let $(N, h)$ is a complete Riemannian manifold with parallel Ricci tensor. If the Ricci curvature has a negative upper bound $-\lambda<0$ and the curvature tensor $R$ and its covariant derivatives of any order have uniform bounds (i.e., $\left.\left|\nabla^{l} R\right| \leq B_{l}, l=0,1,2, \cdots\right)$, then, for any integer $k \geq 3$ the Cauchy problem of (1.4) with the initial value map $u_{0} \in H^{k}\left(S^{1}, N\right)$ admits a local solution $u \in L^{\infty}\left([0, T], H^{k}\left(S^{1}, N\right)\right)$, where $T=T\left(N,\left\|u_{0}\right\|_{H^{3}}\right)$.

Before proving Lemma 2.4, we remark that in [8], Ding and Wang have shown that the $H^{m}$ norm of section $\nabla u$ is equivalent to the usual Sobolev $W^{m+1,2}$ norm of the map $u$. Precisely, we have

Lemma 2.5. ([8]) Assume that $N$ is a compact Riemannian manifold with or without boundary and $m \geq 1$. Then there exists a constant $C=C(N, m)$ such that for all $u \in C^{\infty}\left(S^{1}, N\right)$,

$$
\|D u\|_{W^{m-1,2}} \leq C \sum_{i=1}^{m}\|\nabla u\|_{H^{m-1,2}}^{i}
$$

and

$$
\|\nabla u\|_{H^{m-1,2}} \leq C \sum_{i=1}^{m}\|D u\|_{W^{m-1,2}}^{i} .
$$

Now we turn to the proof of Lemma 2.4. The process goes similar with the proof of Lemma 3.3 in ([27]) which is about the local existence of the KdV geometric flow .

Proof. Assume $N$ is compact and we imbed $N$ into $\mathbb{R}^{n}$. If $u_{0}: S^{1} \rightarrow N$ is $C^{\infty}$, then from Lemma 2.2 we have that the Cauchy problem (2.1) admits a smooth solution $u_{\varepsilon}$ which satisfies the estimates in Lemma 2.2. Hence by Lemma 2.2 and Lemma 2.5, for any integer $p>0$ and $\varepsilon \in(0,1]$ we have:

$$
\sup _{t \in[0, T]}\left\|u_{\varepsilon}\right\|_{W^{p, 2}(N)} \leq C_{p}\left(N, u_{0}\right)
$$


where $C_{p}\left(N, u_{0}\right)$ does not depend on $\varepsilon$. Hence, by sending $\varepsilon \rightarrow 0$ and applying the embedding theorem of Sobolev spaces to $u$, we have $u_{\varepsilon} \rightarrow u \in C^{p}\left(S^{1} \times[0, T]\right)$ for any $p$. It is easy to check that $u$ is a solution to the Cauchy problem (1.4).

If $u_{0}: S^{1} \rightarrow N$ is not $C^{\infty}$, but $u_{0} \in W^{k, 2}\left(S^{1}, N\right)$, we may always select a sequence of $C^{\infty}$ maps $u_{i 0}: S^{1} \rightarrow N,(i=1,2, \cdots, n \cdots)$, such that

$$
u_{i 0} \rightarrow u_{0} \quad \text { in } \quad W^{k, 2}, \quad \text { as } \quad i \rightarrow \infty .
$$

Thus following from Lemma 2.5 we have

$$
\left\|\nabla_{x} u_{i 0}\right\|_{H^{k-1}} \rightarrow\left\|\nabla_{x} u_{0}\right\|_{H^{k-1}}, \quad \text { as } \quad i \rightarrow \infty .
$$

Thus there exists a unique, smooth solution $u_{i}$, defined on time interval $\left[0, T_{i}\right]$, of the Cauchy problem (2.1) with $u_{0}$ replaced by $u_{i 0}$. Furthermore, from Lemma 2.2 we could obtain that if $i$ is large enough, then there exists a uniform positive lower bound of $T_{i}$, denoted by $T$, such that the following inequality holds uniformly with respect to large enough $i$ :

$$
\sup _{t \in[0, T]}\left\|\nabla u_{i}(t)\right\|_{H^{k-1}} \leq C\left(T,\left\|u_{0 x}\right\|_{H^{k-1}}\right) .
$$

Hence from Lemma 2.5 we deduce

$$
\sup _{t \in[0, T]}\left\|D u_{i}(t)\right\|_{W^{k-1,2}} \leq C\left(T,\left\|u_{0 x}\right\|_{W^{k-1,2}}\right),
$$

and by (2.1) we have

$$
\frac{d u_{i}}{d t} \in L^{2}\left([0, T], W^{k-3,2}\left(S^{1}, N\right)\right) .
$$

By Sobolev theorem, it is easy to see that $u_{i} \in C^{0, \frac{1}{2}}\left([0, T], W^{k-3,2}\left(S^{1}, N\right)\right)$.

Interpolating the spaces $L^{\infty}\left([0, T], W^{k, 2}\left(S^{1}, N\right)\right)$ and $C^{0, \frac{1}{2}}\left([0, T], W^{k-3,2}\left(S^{1}, N\right)\right)$ yields that

$$
u_{i} \in C^{0, \gamma}\left([0, T], W^{k-6 \gamma, 2}\left(S^{1}, N\right)\right) \quad \text { for } \quad \gamma \in\left(0, \frac{1}{2}\right) .
$$

Therefore when letting $\gamma$ small while using Rellich's theorem and the Ascoli-Arzela theorem, from (2.22) and (2.23) we obtain that there exists

$$
u \in L^{\infty}\left([0, T], W^{k, 2}\left(S^{1}, N\right)\right) \cap C\left([0, T], W^{k-1,2}\left(S^{1}, N\right)\right)
$$

such that

$$
\begin{aligned}
& u_{i} \rightarrow u \quad\left[\text { weakly }^{*}\right] \quad \text { in } \quad L^{\infty}\left([0, T], W^{k, 2}\left(S^{1}, N\right)\right), \\
& u_{i} \rightarrow u \text { in } C\left([0, T], W^{k-1,2}\left(S^{1}, N\right)\right)
\end{aligned}
$$

upon extracting a subsequence and re-indexing if necessary.

It remains to verify that $u$ is a strong solution to (1.4). We need to check that for any $v \in C^{\infty}\left(S^{1} \times[0, T], \mathbb{R}^{n}\right)$ there holds

$$
\int_{0}^{T} \int_{S^{1}}\left\langle u_{t}, v\right\rangle d x d t=\int_{0}^{T} \int_{S^{1}}\left\langle\nabla_{x}^{2} u_{x}, v\right\rangle d x d t+\rho \int_{0}^{T} \int_{S^{1}}\left\langle\operatorname{Ric}(u)\left(u_{x}, u_{x}\right) u_{x}, v\right\rangle d x d t .
$$


First we always have that for each $u_{i}$

$$
\int_{0}^{T} \int_{S^{1}}\left\langle u_{i t}, v\right\rangle d x d t=\int_{0}^{T} \int_{S^{1}}\left\langle\nabla_{x}^{2} u_{i x}, v\right\rangle d x d t+\rho \int_{0}^{T} \int_{S^{1}}\left\langle\operatorname{Ric}\left(u_{i}\right)\left(u_{i x}, u_{i x}\right) u_{i x}, v\right\rangle d x d t .
$$

For each $y \in N \subset \mathbb{R}^{n}$, let $P(y)$ be the orthogonal projection from $\mathbb{R}^{n}$ onto $T_{y} N$, we have

$$
\begin{aligned}
\nabla_{x} u_{x} & =P(u) u_{x x} \\
\nabla_{x}^{2} u_{x} & =P(u)(P(u))_{x} u_{x x}+P(u) u_{x x x}, \\
\nabla_{x}^{2} u_{i x} & =P\left(u_{i}\right)\left(P\left(u_{i}\right)\right)_{x} u_{i x x}+P\left(u_{i}\right) u_{i x x x} .
\end{aligned}
$$

Hence we have

$$
\begin{aligned}
& \int_{0}^{T} \int_{S^{1}}\left|\left\langle\nabla_{x}^{2} u_{x}, v\right\rangle-\left\langle\nabla_{x}^{2} u_{i x}, v\right\rangle\right| d x d t \\
\leq & \int_{0}^{T} \int_{S^{1}}\left|\left\langle\left(P(u)-P\left(u_{i}\right)\right) u_{x x x}, v\right\rangle\right| d x d t+\int_{0}^{T} \int_{S^{1}}\left|\left\langle P\left(u_{i}\right)\left(u_{x x x}-u_{i x x x}\right), v\right\rangle\right| d x d t \\
\quad & \int_{0}^{T} \int_{S^{1}}\left|\left\langle\left(P(u)(P(u))_{x}-P\left(u_{i}\right)\left(P\left(u_{i}\right)\right)\right)_{x} u_{x x}, v\right\rangle\right| d x d t \\
& +\int_{0}^{T} \int_{S^{1}}\left|\left\langle P\left(u_{i}\right)\left(P\left(u_{i}\right)\right)_{x}\left(u_{x x}-u_{i x x}\right), v\right\rangle\right| d x d t .
\end{aligned}
$$

Moreover,

$$
\begin{aligned}
& \int_{0}^{T} \int_{S^{1}}\left|\left\langle\operatorname{Ric}(u)\left(u_{x}, u_{x}\right) u_{x}, v\right\rangle-\left\langle\operatorname{Ric}\left(u_{i}\right)\left(u_{i x}, u_{i x}\right) u_{i x}, v\right\rangle\right| d x d t \\
\leq & \int_{0}^{T} \int_{S^{1}}\left|\operatorname{Ric}(u)\left(u_{x}, u_{x}\right) u_{x}-\operatorname{Ric}\left(u_{i}\right)\left(u_{i x}, u_{i x}\right) u_{i x}\right||v| d x d t .
\end{aligned}
$$

Since $N$ is compact, it is obviously that

$$
\|P(\cdot) D(P(\cdot))\|_{L^{\infty}(N)}<\infty \mid ; \quad\|\operatorname{Ric}(\cdot)\|_{L^{\infty}(N)}<\infty .
$$

Hence we obtain that each term on the right hand side of (2.26) and (2.27) converges zero as $i$ goes to infinity. This implies that

$$
\begin{aligned}
& \lim _{i \rightarrow \infty} \int_{0}^{T} \int_{S^{1}}\left\langle\nabla_{x}^{2} u_{i x}, v\right\rangle d x d t=\int_{0}^{T} \int_{S^{1}}\left\langle\nabla_{x}^{2} u_{x}, v\right\rangle d x d t \\
& \lim _{i \rightarrow \infty} \int_{0}^{T} \int_{S^{1}}\left\langle\operatorname{Ric}\left(u_{i}\right)\left(u_{i x}, u_{i x}\right) u_{i x}, v\right\rangle d x d t=\int_{0}^{T} \int_{S^{1}}\left\langle\operatorname{Ric}(u)\left(u_{x}, u_{x}\right) u_{x}, v\right\rangle d x d t .
\end{aligned}
$$

On the other hand, we also have

$$
\lim _{i \rightarrow \infty} \int_{0}^{T} \int_{S^{1}}\left\langle u_{i t}, v\right\rangle d x d t=-\int_{0}^{T} \int_{S^{1}}\left\langle u, v_{t}\right\rangle d x d t+\int_{S^{1}}\left(\langle u(T), v(T)\rangle-\left\langle u_{0}, v(0)\right\rangle\right) d x .
$$

Thus, from the above equalities we have

$$
\int_{0}^{T} \int_{S^{1}}\left\langle\nabla_{x}^{2} u_{x}, v\right\rangle d x d t+\rho \int_{0}^{T} \int_{S^{1}}\left\langle\operatorname{Ric}\left(u_{x}, u_{x}\right) u_{x}, v\right\rangle d x d t
$$




$$
=-\int_{0}^{T} \int_{S^{1}}\left\langle u, v_{t}\right\rangle d x d t+\int_{S^{1}}\left(\langle u(T), v(T)\rangle-\left\langle u_{0}, v(0)\right\rangle\right) d x .
$$

Note that $\nabla_{x}^{2} u_{x} \in L^{2}\left(S^{1} \times[0, T], \mathbb{R}^{n}\right)$, thus (2.28) implies $u_{t} \in L^{2}\left(S^{1} \times[0, T], \mathbb{R}^{n}\right)$. Therefore for any smooth function $v$ we always have

$$
\int_{0}^{T} \int_{S^{1}}\left\langle u_{t}, v\right\rangle d x d t=\int_{0}^{T} \int_{S^{1}}\left\langle\nabla_{x}^{2} u_{x}+\rho \operatorname{Ric}\left(u_{x}, u_{x}\right) u_{x}, v\right\rangle d x d t
$$

which means that $u$ is a strong solution of (2.1).

It is easy to see that if $N$ is a noncompact manifold with bounded geometry and the domain is $S^{1}$, we could find a compact subset of $N$, denoted by $\Omega$, such that $u_{0}\left(S^{1}\right) \subset \Omega \subset \mathbb{R}^{n}$. Therefore we could repeat the same process as in the case $N$ is compact then we obtain the same results and complete the proof.

Now we could show the uniqueness of the solutions and prove Theorem 1.1.

Proof of Theorem 1.1. Without loss of generality, we always assume that $N$ is compact, since $u(x, t) \in L^{\infty}\left([0, T], H^{4}\left(S^{1}, N\right)\right)$ implies that $\left\{u(x, t):(x, t) \in S^{1} \times[0, T]\right\} \subset \subset N$. We regard $N$ as a submanifold of $\mathbb{R}^{n}$. Let $u, v: S^{1} \times[0, T] \rightarrow N \subset \mathbb{R}^{n}$ be two solutions of (1.4) such that $u(x, 0)=v(x, 0)=u_{0}$ and $u, v \in L^{\infty}\left([0, T], W^{k, 2}\left(S^{1}, N\right)\right)$ for $k \geq 4$. Let $w=u-v$ which makes sense as a $\mathbb{R}^{n}$-valued function. It is worthy to point out that the Ricci curvature Ric here should be regarded as operators on $\mathbb{R}^{n}$, such that $\operatorname{Ric}(u)\left(u_{x}, u_{x}\right) u_{x}-\operatorname{Ric}(v)\left(v_{x}, v_{x}\right) v_{x}$ makes sense in $\mathbb{R}^{n}$.

From (2.24) we have that

$$
\nabla_{x}^{2} u_{x}=P(u) u_{x x x}+P(u)(P(u))_{x} u_{x x} .
$$

Thus

$$
u_{t}=P(u) u_{x x x}+P(u)(P(u))_{x} u_{x x}+\rho \operatorname{Ric}(u)\left(u_{x}, u_{x}\right) u_{x} .
$$

Hence we have

$$
\begin{aligned}
w_{t}= & P(u) w_{x x x}+[P(u)-P(v)] v_{x x x} \\
& +P(u)(P(u))_{x} w_{x x}+\left(P(u)(P(u))_{x}-P(v)(P(v))_{x}\right) v_{x x} \\
& +\rho\left(\operatorname{Ric}(u)\left(u_{x}, u_{x}\right) u_{x}-\operatorname{Ric}(v)\left(v_{x}, v_{x}\right) v_{x}\right) .
\end{aligned}
$$

We could proove that there exists a constant $C$ which depends only on $N$ and $\|u\|_{W^{4,2}}$ and $\|v\|_{W^{4,2}}$ such that

$$
\frac{d}{d t}\|w\|_{W^{1,2}}^{2} \leq C\|w\|_{W^{1,2}}^{2},
$$

then by Gronwall's inequality we could obtain that $w \equiv 0$ and obtain the uniqueness of the solutions. To see this, we start by calculating

$$
\frac{1}{2} \frac{d}{d t} \int\left|w_{x}\right|^{2} d x=-\int\left\langle w_{x x}, w_{t}\right\rangle d x
$$




$$
\begin{aligned}
= & -\int\left\langle w_{x x}, P(u) w_{x x x}\right\rangle d x-\int\left\langle w_{x x},[P(u)-P(v)] v_{x x x}\right\rangle d x \\
& -\int\left\langle w_{x x}, P(u)(P(u))_{x} w_{x x}\right\rangle d x-\int\left\langle w_{x x},\left[P(u)(P(u))_{x}-P(v)(P(v))_{x}\right] v_{x x}\right\rangle d x \\
& -\rho \int\left\langle w_{x x},\left(\operatorname{Ric}(u)\left(u_{x}, u_{x}\right) u_{x}-\operatorname{Ric}(v)\left(v_{x}, v_{x}\right) v_{x}\right)\right\rangle d x .
\end{aligned}
$$

Then, similar with the process in [27], for the first four terms of (2.31), we have :

$$
\begin{aligned}
& -\int\left\langle w_{x x}, P(u) w_{x x x}\right\rangle d x-\int\left\langle w_{x x},[P(u)-P(v)] v_{x x x}\right\rangle d x \\
& -\int\left\langle w_{x x}, P(u)(P(u))_{x} w_{x x}\right\rangle d x-\int\left\langle w_{x x},\left[P(u)(P(u))_{x}-P(v)(P(v))_{x}\right] v_{x x}\right\rangle d x \\
\leq & C\left(\int\left|w_{x}\right|^{2} d x+\int\left|w_{x}\right||w| d x+\left.|| w\right|_{L^{\infty}} \int\left(\left|w_{x}\right|+|w|\right)\left|v_{x x x}\right| d x+\int\left|w_{x}\right|^{2}\left|v_{x x x}\right| d x\right) \\
\leq & C\|w\|_{W^{1,2}}^{2},
\end{aligned}
$$

where $C$ depends on $N,\|u\|_{W^{3,2}}$ and $\|v\|_{W^{4,2}}$. The calculations about these estimates are same with that in $[27]$ and we omit the details here. For the last term of (2.31), we have

$$
\begin{aligned}
& -\frac{1}{2} \int\left\langle w_{x x},\left(\operatorname{Ric}(u)\left(u_{x}, u_{x}\right) u_{x}-\operatorname{Ric}(v)\left(v_{x}, v_{x}\right) v_{x}\right)\right\rangle d x \\
= & -\frac{1}{2} \int \operatorname{Ric}(u)\left(u_{x}, u_{x}\right)\left\langle w_{x x}, w_{x}\right\rangle d x \\
& -\frac{1}{2} \int\left(\operatorname{Ric}(u)\left(u_{x}, u_{x}\right)-\operatorname{Ric}(v)\left(v_{x}, v_{x}\right)\right)\left\langle w_{x x}, v_{x}\right\rangle d x \\
= & \frac{1}{4} \int D_{x}\left(\operatorname{Ric}(u)\left(u_{x}, u_{x}\right)\right)\left\langle w_{x}, w_{x}\right\rangle d x \\
& +\frac{1}{2} \int\left(\operatorname{Ric}(u)\left(u_{x}, u_{x}\right)-\operatorname{Ric}(v)\left(v_{x}, v_{x}\right)\right)_{x}\left\langle w_{x}, v_{x}\right\rangle d x \\
& +\frac{1}{2} \int\left(\operatorname{Ric}(u)\left(u_{x}, u_{x}\right)-\operatorname{Ric}(v)\left(v_{x}, v_{x}\right)\right)\left\langle w_{x}, v_{x x}\right\rangle d x \\
\leq & C\left(\int\left|w_{x}\right|^{2} d x+\int\left|w_{x}\left\|v_{x}\left|d x+\int\right| w_{x}\right\| v_{x x}\right| d x\right) \\
\leq & C\|w\|_{W^{1,2}}^{2},
\end{aligned}
$$

where $C$ depends on $N,\|u\|_{W^{3,2}}$ and $\|v\|_{W^{3,2}}$.

Combining (2.32) and (2.33) yields

$$
\frac{d}{d t} \int\left|w_{x}\right|^{2} d x \leq C|| w \|_{W^{1,2}}^{2}
$$

where $C$ depends on $N,\|u\|_{W^{3,2}}$ and $\|v\|_{W^{4,2}}$.

Moreover, by a similar argument we could obtain that

$$
\frac{d}{d t} \int|w|^{2} d x \leq C\|w\|_{W^{1,2}}^{2}
$$


where $C$ depends on $N,\|u\|_{W^{3,2}}$ and $\|v\|_{W^{3,2}}$. We omit the detail.

Hence we have

$$
\frac{d}{d t}\|w\|_{W^{1,2}}^{2} \leq C\|w\|_{W^{1,2}}^{2}
$$

where $C$ depends on $N,\|u\|_{W^{4,2}}$ and $\|v\|_{W^{4,2}}$. This implies that $w \equiv 0$ since $w(x, 0)=0$, i.e. the solution is unique.

Thus it suffices to show that $\nabla_{x}^{k-1} u_{x} \in C\left([0, T] ; L^{2}\left(S^{1}, T N\right)\right)$ for $k \geq 4$. In the proof of Lemma 2.4 we have seen that the solution $u \in L^{\infty}\left([0, T], H^{k}\left(S^{1}, N\right)\right) \cap C\left([0, T], H^{k-1}\left(S^{1}, N\right)\right)$, thus by the discussion about (2.21), (2.22) and the equation of the new geometric flow, we could easily get that

$$
\frac{d}{d t}\left\|\nabla_{x}^{k-1} u_{x}\right\|_{L^{2}}^{2} \leq C
$$

which implies that

$$
\left\|\nabla_{x}^{k-1} u_{x}(t, x)\right\|_{L^{2}\left(S^{1}, T N\right)}^{2} \leq\left\|\nabla_{x}^{k-1} u_{x}(0, x)\right\|_{L^{2}\left(S^{1}, T N\right)}^{2}+C t .
$$

Hence we obtain

$$
\lim _{t \rightarrow 0} \sup \left\|\nabla_{x}^{k-1} u_{x}(t, x)\right\|_{L^{2}\left(S^{1}, T N\right)}^{2} \leq\left\|\nabla_{x}^{k-1} u_{x}(0, x)\right\|_{L^{2}\left(S^{1}, T N\right)}^{2} .
$$

On the other hand, $u \in L^{\infty}\left([0, T], H^{k}\left(S^{1}, N\right)\right) \cap C\left([0, T], H^{k-1}\left(S^{1}, N\right)\right)$ implies that, with respect to $t, \nabla_{x}^{k-1} u_{x}(t, x)$ is weakly continuous in $L^{2}\left(S^{1}, T N\right)$, we have

$$
\left\|\nabla_{x}^{k-1} u_{x}(0, x)\right\|_{L^{2}\left(S^{1}, T N\right)}^{2} \leq \liminf _{t \rightarrow 0}\left\|\nabla_{x}^{k-1} u_{x}(t, x)\right\|_{L^{2}\left(S^{1}, T N\right)}^{2} .
$$

Thus,

$$
\lim _{t \rightarrow 0}\left\|\nabla_{x}^{k-1} u_{x}(t, x)\right\|_{L^{2}}^{2}=\left\|\nabla_{x}^{k-1} u_{x}(0, x)\right\|_{L^{2}}^{2}
$$

which implies that $\nabla_{x}^{k-1} u_{x}(t, x)$ is continuous in $L^{2}\left(S^{1}, T N\right)$ at $t=0$. Now by the uniqueness of $u(t, x)$, we get that $\nabla_{x}^{k-1} u_{x}(t, x)$ is continuous at each $t \in[0, T]$, i.e. $u \in C\left([0, T], H^{k}\left(S^{1}, N\right)\right)$ for all $k \geq 4$. Thus we complete the proof of Theorem 1.1. However, if $k \leq 3$, we could not get the continuity of $\|u\|_{H^{k}}$ about $t$ on $[0, T]$ without the uniqueness of the solutions.

We are now ready to proof Theorem 1.2.

Proof. We only discuss the case that Ric $\leq-\lambda<0$ here. For Ric $\geq \lambda>0$, the process is easier. To show the existence of the Cauchy problem (1.4) with an initial map $u_{0} \in H^{4}\left(S^{1}, N\right)$, we first consider the following Cauchy problems:

$$
\left\{\begin{array}{l}
u_{t}=\nabla_{x}^{2} u_{x}+\rho \operatorname{Ric}\left(u_{x}, u_{x}\right) u_{x}, \quad x \in S^{1} ; \\
u(x, 0)=u_{0}^{i}(x) .
\end{array}\right.
$$

Here $u_{0}^{i} \in C^{\infty}\left(S^{1}, N\right)$ and $\left\|u_{0}^{i}-u_{0}\right\|_{H^{4}} \rightarrow 0$. By (ii) in Lemma 2.2 we know that for each $i$ and any $k \geq 5,(2.36)$ admits a local solution $u^{i} \in L^{\infty}\left(\left[0, T_{i}^{\max }\right), H^{k}\left(S^{1}, N\right)\right)$, where $T_{i}^{\max }=$ $T_{i}^{\max }\left(S^{1},\left\|u_{0}^{i}\right\|_{H^{5}}\right)$ is the maximal existence interval of $u^{i}$. 
As $N$ may not be compact, we let $\Omega_{i} \triangleq\left\{p \in N: \operatorname{dist}_{N}\left(p, u_{0}^{i}\left(S^{1}\right)\right)<1\right\}$, which is an open subset of $N$ with compact closure $\bar{\Omega}_{i}$. Denote

$$
\Omega_{\infty} \triangleq\left\{p \in N: \operatorname{dist}_{N}\left(p, u_{0}\left(S^{1}\right)\right)<1\right\} \quad \text { and } \quad \Omega_{0} \triangleq\left\{p \in N: \operatorname{dist}_{N}\left(p, \Omega_{\infty}\right)<1\right\} .
$$

Since $\left\|u_{0}^{i}-u_{0}\right\|_{H^{4}} \rightarrow 0$, then $\Omega_{i} \subset \subset \Omega_{0}$ as $i$ is large enough. Let

$$
T_{i}^{\prime}=\sup \left\{t>0: u^{i}\left(S^{1}, t\right) \subset \Omega_{i}\right\} .
$$

By the same argument as in Lemma 2.2 we can show that there holds true for all $t \in\left[0, T_{i}\right]$

$$
\frac{d}{d t}\left(-\frac{1}{\lambda} \sum_{s=0}^{2} \int \operatorname{Ric}\left(\nabla_{x}^{s} u_{x}^{i}, \nabla_{x}^{s} u_{x}^{i}\right) d x\right) \leq C\left(\Omega_{0}, \lambda\right) \sum_{l=2}^{4}\left(-\frac{1}{\lambda} \sum_{s=0}^{2} \int \operatorname{Ric}\left(\nabla_{x}^{s} u_{x}^{i}, \nabla_{x}^{s} u_{x}^{i}\right) d x\right)^{l} .
$$

If we let $f^{i}(t)=-\frac{1}{\lambda} \sum_{s=0}^{2} \int \operatorname{Ric}\left(\nabla_{x}^{s} u_{x}^{i}, \nabla_{x}^{s} u_{x}^{i}\right) d x+1$ and $g^{i}(t)=\left\|u_{x}^{i}\right\|_{H^{2}}^{2}+1$, then we have

$$
\frac{d f^{i}}{d t} \leq C\left(\Omega_{0}\right)\left(f^{i}\right)^{4}
$$

Moreover, since $\Omega_{i} \subset \subset \Omega_{0}$ and $\overline{\Omega_{0}}$ is compact, we have

$$
f^{i}(0)=-\frac{1}{\lambda} \sum_{s=0}^{2} \int \operatorname{Ric}\left(\nabla_{x}^{s} u_{0 x}^{i}, \nabla_{x}^{s} u_{0 x}^{i}\right) d x+1 \leq C\left(\Omega_{0}, \lambda\right)\left\|u_{0 x}^{i}\right\|_{H^{2}}^{2}+1 .
$$

It follows from the above differential inequality (2.37) that there holds true

$$
g^{i}(t) \leq f^{i}(t) \leq\left(\frac{\left(f^{i}(0)\right)^{3}}{1-3\left(f^{i}(0)\right)^{3} C\left(\Omega_{0}, \lambda\right) t}\right)^{\frac{1}{3}},
$$

as

$$
t<\frac{1}{3\left(f^{i}(0)\right)^{3} C\left(\Omega_{0}, \lambda\right)} .
$$

Then, there exists constants

$$
\begin{aligned}
0<T_{0}^{i} & =T_{0}^{i}\left(\Omega_{0},\left\|u_{0 x}\right\|_{H^{2}}, \lambda\right) \\
& =\frac{1}{4\left(\lambda\left\|u_{0 x}\right\|_{H^{2}}^{2}+1\right)^{3} C\left(\Omega_{0}, \lambda\right)} \leq \frac{1}{4\left(f^{i}(0)\right)^{3} C\left(\Omega_{0}, \lambda\right)}
\end{aligned}
$$

and $C_{0}^{i}=4^{\frac{1}{3}} f^{i}(0)>0$ such that

$$
\lambda\left\|u_{x}^{i}\right\|_{H^{2}}^{2} \leq \sum_{s=0}^{2} \int \operatorname{Ric}\left(\nabla_{x}^{s} u_{0 x}^{i}, \nabla_{x}^{s} u_{0 x}^{i}\right) d x \leq C_{0}^{i} \leq \widetilde{C}_{0}^{i}, \quad t \in\left[0, \min \left(T_{0}^{i}, T_{i}^{\prime}\right)\right],
$$

where $\widetilde{C}_{0}^{i}=4^{\frac{1}{3}}\left(C\left(\Omega_{0}, \lambda\right)\left\|u_{0 x}^{i}\right\|_{H^{2}}^{2}+1\right)$.

For $k \geq 3$, there exists

$$
0<C_{k-2}^{i}=C_{k-2}\left(\lambda,-\frac{1}{\lambda} \sum_{s=0}^{k} \int \operatorname{Ric}\left(\nabla_{x}^{s} u_{0 x}^{i}, \nabla_{x}^{s} u_{0 x}^{i}\right) d x\right) \leq \widetilde{C}_{k-2}\left(\lambda, \Omega_{0},\left\|u_{0 x}^{i}\right\|_{H^{2}}\right)
$$


such that for $t \in\left[0, \min \left(T_{0}^{i}, T_{i}^{\prime}\right)\right]$

$$
\left\|u_{x}^{i}\right\|_{H^{k}} \leq-\frac{1}{\lambda} \sum_{s=0}^{k} \int \operatorname{Ric}\left(\nabla_{x}^{s} u_{0 x}^{i}, \nabla_{x}^{s} u_{0 x}^{i}\right) d x \leq C_{k-2}^{i} \leq \widetilde{C}_{k-2}\left(\lambda, \Omega_{0},\left\|u_{0 x}^{i}\right\|_{H^{2}}\right) .
$$

Since $\left\|u_{0}^{i}-u_{0}\right\|_{H^{4}} \rightarrow 0$, when $i$ is large enough we have

$$
T_{0}=\frac{1}{4\left(\left\|u_{0 x}\right\|_{H^{2}}^{2}+1+\delta_{0}\right) C\left(\Omega_{0}\right)}<T_{0}^{i},
$$

where $\delta_{0}$ is a small positive number. It is easy to see that, as $i$ is large enough,

$$
\widetilde{C}_{0}^{i} \leq \widetilde{C}_{0}\left(\left\|u_{0 x}\right\|_{H^{2}}\right)+\delta_{0} \quad \text { and } \quad \widetilde{C}_{1}^{i} \leq \widetilde{C}_{1}\left(\left\|u_{0 x}\right\|_{H^{3}}\right)+\delta_{0} .
$$

Note that we always have $T_{i}^{\max }>\min \left(T_{0}, T_{i}^{\prime}\right)$ when $i$ is large enough. Otherwise, by Lemma 2.4 we can find a time-local solution $u_{1}$ of (1.4) and $u_{1}$ satisfies the initial value condition

$$
u_{1}\left(x, T_{i}^{\max }-\epsilon\right)=u\left(x, T_{i}^{\max }-\epsilon\right),
$$

where $0<\epsilon<T_{i}^{\max }$ is a small number. Then by the local existence theorem, $u_{1}$ exists on the time interval $\left(T_{i}^{\max }-\epsilon, T_{i}^{\max }-\epsilon+\eta\right)$ for some constant $\eta>0$. The uniform bounds on $\left\|u_{x}\right\|_{H^{2}}$ and $\left\|\nabla_{x}^{m} u_{x}\right\|_{L^{2}}$ (for all $m>2$ ) implies that $\eta$ is independent of $\epsilon$. Thus, by choosing $\epsilon$ sufficiently small, we have

$$
T_{i}^{e}=T_{i}^{\max }-\epsilon+\eta>T_{i}^{\max } .
$$

By the uniqueness result, we have that $u_{1}(x, t)=u(x, t)$ for all $t \in\left[T_{i}^{\max }-\epsilon, T_{i}^{e}\right)$. Thus we get a solution of the Cauchy problem (1.4) on the time interval $\left[0, T_{e}\right)$, which contradicts the maximality of $T_{i}^{\max }$.

Now we need to show that $T_{i}^{\prime}$ have a uniform lower bound as $i$ is large enough. For each large enough $i$, if $T_{i}^{\prime} \geq T_{0}$ we obtain the lower bound. Otherwise, by the same argument as in Lemma 2.2 we have

$$
T_{i}^{\prime} \geq \frac{1}{\mathcal{M}_{i}}
$$

where

$$
\mathcal{M}_{i}=\sup _{\left[0, \min \left(T_{0}, T_{i}^{\prime}\right)\right]}\left\|u_{t}^{i}\right\|_{L^{\infty}} \leq C \sup _{\left[0, \min \left(T_{0}, T_{i}^{\prime}\right)\right]}\left\|u_{t}^{i}\right\|_{H^{1}}^{a} \sup _{\left[0, \min \left(T_{0}, T_{i}^{\prime}\right)\right]}\left\|u_{t}^{i}\right\|_{L^{2}}^{1-a} \equiv M_{i} .
$$

It should be pointed out that to derive the estimates $L^{\infty}$ estimates on $\left\|u_{t}^{i}(s)\right\|_{L^{2}}$ and $\left\|u_{t}^{i}(s)\right\|_{H^{1}}$ we need only to have $u^{i} \in L^{\infty}\left(\left[0, \min \left(T_{0}, T_{i}^{\prime}\right)\right], H^{4}\left(S^{1}, N\right)\right)$, since the equation of the geometric flow (1.4) is a third-order dispersive equation. It is not difficult to see from (2.38) that there exists a positive constant $M\left(\Omega_{0},\left\|u_{0 x}\right\|_{H^{3}}\right)$ such that, as $i$ is large enough,

$$
M_{i}\left(\Omega_{0},\left\|u_{0 x}^{i}\right\|_{H^{3}}\right) \leq M\left(\Omega_{0},\left\|u_{0 x}\right\|_{H^{3}}\right),
$$

since $\left\|u_{0}^{i}-u_{0}\right\|_{H^{4}} \rightarrow 0$. 
Let $T^{*}=\min \left(T_{0}, \frac{1}{M}\right)$. As $i$ is large enough, we always have $u^{i} \in L^{\infty}\left(\left[0, T^{*}\right], H^{4}\left(S^{1}, N\right)\right)$. By letting $i \rightarrow \infty$ and taking the same arguments as in Lemma 2.4, we know there exists $u \in L^{\infty}\left(\left[0, T^{*}\right], H^{4}\left(S^{1}, N\right)\right)$ such that

$$
u^{i} \rightarrow u \quad\left[\text { weakly }^{*}\right] \quad \text { in } \quad L^{\infty}\left(\left[0, T^{*}\right], H^{4}\left(S^{1}, N\right)\right)
$$

and $u$ is a local solution to (1.4). Theorem 1.1 guarantees that the local solution is unique and it is continuous with respect to $t$, i.e., $u \in C\left(\left[0, T^{*}\right], H^{4}\left(S^{1}, N\right)\right)$. Thus, we finish the proof of the theorem.

\section{Conversation Laws}

In this section, we let $(N, h)$ be a locally symmetric space with metric $h$. Then it is easy to see that $\nabla R i c \equiv 0$ on $N$ since $\nabla R \equiv 0$. Then for a smooth solution $u(x, t): S^{1} \times(0, T) \rightarrow N$ of the Cauchy problem (1.4), we will derive in this section the conservation laws $E_{1}(u), E_{2}(u)$ and the semi-conservation law $E_{3}(u)$ introduced in Sec.1.

Precisely, we have the following results:

Lemma 3.1. Assume $N$ is a locally symmetric space. If $u: S^{1} \times(0, T) \rightarrow N$ is a smooth solution of the Cauchy problem of the new geometric flow (1.4), then

$$
\frac{d E_{1}}{d t}=\frac{d}{d t} \int \operatorname{Ric}\left(u_{x}, u_{x}\right) d x=0,
$$

in other words, $E_{1}(u)=E_{1}\left(u_{0}\right)$ for all $t \in(0, T)$.

Proof. With the assumption of $N$, we have $\nabla R i c=0$. Hence

$$
\begin{aligned}
\frac{d E_{1}}{d t} & =\frac{d}{d t} \int \operatorname{Ric}\left(u_{x}, u_{x}\right) d x=2 \int \operatorname{Ric}\left(\nabla_{t} u_{x}, u_{x}\right) \\
& =2 \int \operatorname{Ric}\left(\nabla_{x} u_{t}, u_{x}\right) d x=-2 \int \operatorname{Ric}\left(\nabla_{x} u_{x}, u_{t}\right) .
\end{aligned}
$$

Substituting (1.4) into above yields

$$
\begin{aligned}
\frac{d E_{1}}{d t} & =-2 \int \operatorname{Ric}\left(\nabla_{x} u_{x}, \nabla_{x}^{2} u_{x}\right) d x-2 \rho \int \operatorname{Ric}\left(\nabla_{x} u_{x}, u_{x}\right) \operatorname{Ric}\left(u_{x}, u_{x}\right) d x \\
& =-\int \nabla_{x}\left(\operatorname{Ric}\left(\nabla_{x} u_{x}, \nabla_{x} u_{x}\right)\right)-\frac{\rho}{2} \int \nabla_{x}\left(\operatorname{Ric}\left(u_{x}, u_{x}\right)^{2}\right) \\
& =0 .
\end{aligned}
$$

This completes the proof.

Lemma 3.2. Assume $N$ is a locally symmetric space. If $u: S^{1} \times(0, T) \rightarrow N$ is a smooth solution of the Cauchy problem of the new geometric flow (1.4) and let

$$
E_{2}(u)=\int \operatorname{Ric}\left(\nabla_{x} u_{x}, \nabla_{x} u_{x}\right) d x-\frac{\rho}{2} \int \operatorname{Ric}\left(u_{x}, u_{x}\right)^{2} d x .
$$

Then $E_{2}(u)$ is conserved and we have

$$
\frac{d}{d t} E_{2}(u)=0
$$


Proof. We start by differentiating each term of $E_{2}(u)$ with respect to $t$. For the first term of $E_{2}$, after integrating by parts, we have

$$
\begin{aligned}
& \frac{d}{d t} \int \operatorname{Ric}\left(\nabla_{x} u_{x}, \nabla_{x} u_{x}\right) d x=2 \int \operatorname{Ric}\left(\nabla_{t} \nabla_{x} u_{x}, \nabla_{x} u_{x}\right) d x \\
= & 2 \int \operatorname{Ric}\left(\nabla_{x} \nabla_{t} u_{x}, \nabla_{x} u_{x}\right) d x+2 \int \operatorname{Ric}\left(R\left(u_{t}, u_{x}\right) u_{x}, \nabla_{x} u_{x}\right) d x \\
= & 2 \int \operatorname{Ric}\left(\nabla_{x}^{2} u_{t}, \nabla_{x} u_{x}\right) d x+2 \int \operatorname{Ric}\left(R\left(u_{t}, u_{x}\right) u_{x}, \nabla_{x} u_{x}\right) d x \\
= & 2 \int \operatorname{Ric}\left(\nabla_{x}^{3} u_{x}, u_{t}\right) d x+2 \int \operatorname{Ric}\left(u_{t}, R\left(\nabla_{x} u_{x}, u_{x}\right) u_{x}\right) d x .
\end{aligned}
$$

Substituting the equation (1.4) we get

$$
\begin{aligned}
& \frac{d}{d t} \int \operatorname{Ric}\left(\nabla_{x} u_{x}, \nabla_{x} u_{x}\right) d x \\
= & 2 \int \operatorname{Ric}\left(\nabla_{x}^{3} u_{x}, \nabla_{x}^{2} u_{x}\right) d x+2 \int \operatorname{Ric}\left(\nabla_{x}^{2} u_{x}, R\left(\nabla_{x} u_{x}, u_{x}\right) u_{x}\right) d x \\
& +2 \rho \int \operatorname{Ric}\left(\operatorname{Ric}\left(u_{x}, u_{x}\right) u_{x}, R\left(\nabla_{x} u_{x}, u_{x}\right) u_{x}\right) d x \\
& +2 \rho \int \operatorname{Ric}\left(\nabla_{x}^{3} u_{x}, u_{x}\right) \operatorname{Ric}\left(u_{x}, u_{x}\right) d x .
\end{aligned}
$$

The first three terms of right hand side of (3.1) vanish. In fact

$$
2 \int \operatorname{Ric}\left(\nabla_{x}^{3} u_{x}, \nabla_{x}^{2} u_{x}\right) d x=\int \nabla_{x}\left(\operatorname{Ric}\left(\nabla_{x}^{2} u_{x}, \nabla_{x}^{2} u_{x}\right)\right) d x=0
$$

and

$$
\int \operatorname{Ric}\left(\nabla_{x}^{2} u_{x}, R\left(\nabla_{x} u_{x}, u_{x}\right) u_{x}\right) d x=\frac{1}{2} \int \nabla_{x}\left(\operatorname{Ric}\left(\nabla_{x} u_{x}, R\left(\nabla_{x} u_{x}, u_{x}\right) u_{x}\right)\right) d x=0
$$

since $\nabla_{x}$ Ric $=\nabla_{x} R=0$ and $R\left(\nabla_{x} u_{x}, \nabla_{x} u_{x}\right)=0$.

For the third term of (3.1), by the property of Ricci curvature we obtained before, we have

$$
\begin{aligned}
& \int \operatorname{Ric}\left(\operatorname{Ric}\left(u_{x}, u_{x}\right) u_{x}, R\left(\nabla_{x} u_{x}, u_{x}\right) u_{x}\right) d x \\
= & \int \operatorname{Ric}\left(u_{x}, R\left(\nabla_{x} u_{x}, u_{x}\right) u_{x}\right) \cdot \operatorname{Ric}\left(u_{x}, u_{x}\right) d x=0 .
\end{aligned}
$$

Thus, for the last term of (3.1), integrating by parts yields

$$
\begin{aligned}
& \frac{d}{d t} \int \operatorname{Ric}\left(\nabla_{x} u_{x}, \nabla_{x} u_{x}\right) d x=2 \rho \int \operatorname{Ric}\left(\nabla_{x}^{3} u_{x}, u_{x}\right) \operatorname{Ric}\left(u_{x}, u_{x}\right) d x \\
= & -2 \rho \int \operatorname{Ric}\left(\nabla_{x}^{2} u_{x}, \nabla_{x} u_{x}\right) \operatorname{Ric}\left(u_{x}, u_{x}\right) d x-4 \rho \int \operatorname{Ric}\left(\nabla_{x}^{2} u_{x}, u_{x}\right) \operatorname{Ric}\left(\nabla_{x} u_{x}, u_{x}\right) d x \\
= & 6 \rho \int \operatorname{Ric}\left(\nabla_{x} u_{x}, \nabla_{x} u_{x}\right) \operatorname{Ric}\left(\nabla_{x} u_{x}, u_{x}\right) d x
\end{aligned}
$$


Now we consider the second term of $E_{2}$ and differentiate it with respect to $t$

$$
\begin{aligned}
& \frac{d}{d t} \int \operatorname{Ric}\left(u_{x}, u_{x}\right)^{2} d x \\
= & 4 \int \operatorname{Ric}\left(\nabla_{t} u_{x}, u_{x}\right) \operatorname{Ric}\left(u_{x}, u_{x}\right) d x=4 \int \operatorname{Ric}\left(\nabla_{x} u_{t}, u_{x}\right) \operatorname{Ric}\left(u_{x}, u_{x}\right) d x \\
= & -4 \int \operatorname{Ric}\left(u_{t}, \nabla_{x} u_{x}\right) \operatorname{Ric}\left(u_{x}, u_{x}\right) d x-8 \int \operatorname{Ric}\left(u_{t}, u_{x}\right) \operatorname{Ric}\left(\nabla_{x} u_{x}, u_{x}\right) d x .
\end{aligned}
$$

Substituting (1.4) yields

$$
\begin{aligned}
& -\frac{\rho}{2} \frac{d}{d t} \int \operatorname{Ric}\left(u_{x}, u_{x}\right)^{2} d x \\
= & 2 \rho \int \operatorname{Ric}\left(\nabla_{x}^{2} u_{x}, \nabla_{x} u_{x}\right) \operatorname{Ric}\left(u_{x}, u_{x}\right) d x+4 \rho \int \operatorname{Ric}\left(\nabla_{x}^{2} u_{x}, u_{x}\right) \operatorname{Ric}\left(\nabla_{x} u_{x}, u_{x}\right) d x \\
& +6 \rho^{2} \int \operatorname{Ric}\left(u_{x}, \nabla_{x} u_{x}\right)\left|\operatorname{Ric}\left(u_{x}, u_{x}\right)\right|^{2} d x .
\end{aligned}
$$

Note that after integrating by parts we have

$$
\begin{aligned}
& 2 \rho \int \operatorname{Ric}\left(\nabla_{x}^{2} u_{x}, \nabla_{x} u_{x}\right) \operatorname{Ric}\left(u_{x}, u_{x}\right) d x=-2 \rho \int \operatorname{Ric}\left(\nabla_{x} u_{x}, \nabla_{x} u_{x}\right) \operatorname{Ric}\left(\nabla_{x} u_{x}, u_{x}\right) d x \\
= & 2 \rho \int \operatorname{Ric}\left(\nabla_{x}^{2} u_{x}, u_{x}\right) \operatorname{Ric}\left(\nabla_{x} u_{x}, u_{x}\right) d x
\end{aligned}
$$

while

$$
6 \rho^{2} \int \operatorname{Ric}\left(u_{x}, \nabla_{x} u_{x}\right)\left|\operatorname{Ric}\left(u_{x}, u_{x}\right)\right|^{2} d x=\rho^{2} \int \nabla_{x}\left(\operatorname{Ric}\left(u_{x}, u_{x}\right)^{3}\right) d x=0 .
$$

This together with (3.3) yields

$$
-\frac{\rho}{2} \frac{d}{d t} \int \operatorname{Ric}\left(u_{x}, u_{x}\right)^{2} d x=-6 \rho \int \operatorname{Ric}\left(\nabla_{x} u_{x}, \nabla_{x} u_{x}\right) \operatorname{Ric}\left(\nabla_{x} u_{x}, u_{x}\right) d x .
$$

Combining (3.3) and (3.4) we have

$$
\frac{d E_{2}}{d t}=\frac{d}{d t} \int \operatorname{Ric}\left(\nabla_{x} u_{x}, \nabla_{x} u_{x}\right) d x-\frac{\rho}{2} \frac{d}{d t} \int \operatorname{Ric}\left(u_{x}, u_{x}\right)^{2} d x=0 .
$$

This completes the proof.

Remark 2. It is worthy to point out that the two conservation laws hold with the assumption that $N$ is a locally symmetric space. We do not need the condition that the Ricci curvature on $N$ has a positive lower bound or a negative upper bound. However, this condition is required such that the following semi-conservation law would hold true.

Precisely, we have 
Lemma 3.3. Assume $N$ is a locally symmetric space and the Ricci curvature on $N$ has a positive lower bound $\lambda>0$ (or a negative upper bound $-\lambda<0$ ). If $u: S^{1} \times(0, T) \rightarrow N$ is a smooth solution of the Cauchy problem of the new geometric flow (1.4) and let

$$
\begin{aligned}
& E_{3}(u)=6 \int \operatorname{Ric}\left(\nabla_{x}^{2} u_{x}, \nabla_{x}^{2} u_{x}\right) d x-20 \rho \int \operatorname{Ric}\left(\nabla_{x} u_{x}, u_{x}\right)^{2} d x \\
& -10 \rho \int \operatorname{Ric}\left(\nabla_{x} u_{x}, \nabla_{x} u_{x}\right) \operatorname{Ric}\left(u_{x}, u_{x}\right) d x-4 \int \operatorname{Ric}\left(\nabla_{x} u_{x}, R\left(\nabla_{x} u_{x}, u_{x}\right) u_{x}\right) d x .
\end{aligned}
$$

Then we have

$$
\frac{d E_{3}}{d t} \leq C\left(E_{3}+1\right),
$$

where $C$ is a constant depends on $N, \lambda, E_{1}\left(u_{0}\right)$ and $\left\|\nabla_{x} u_{x}\right\|_{L^{2}}$.

Proof. For simplicity, we denote

$$
E_{3}(u)=A_{1} F_{1}+A_{2} F_{2}+A_{3} F_{3}+A_{4} F_{4},
$$

where $A_{1}=6, A_{2}=-20 \rho, A_{3}=-10 \rho, A_{4}=-4$ and

$$
\begin{aligned}
F_{1} & =\int \operatorname{Ric}\left(\nabla_{x}^{2} u_{x}, \nabla_{x}^{2} u_{x}\right) d x \\
F_{2} & =\int \operatorname{Ric}\left(\nabla_{x} u_{x}, u_{x}\right)^{2} d x ; \\
F_{3} & =\int \operatorname{Ric}\left(\nabla_{x} u_{x}, \nabla_{x} u_{x}\right) \operatorname{Ric}\left(u_{x}, u_{x}\right) d x ; \\
F_{4} & =\int \operatorname{Ric}\left(\nabla_{x} u_{x}, R\left(\nabla_{x} u_{x}, u_{x}\right) u_{x}\right) d x .
\end{aligned}
$$

To begin with, we calculate $\frac{d}{d t} F_{1}$ :

$$
\begin{aligned}
& \frac{d}{d t} F_{1}=\frac{d}{d t} \int \operatorname{Ric}\left(\nabla_{x}^{2} u_{x}, \nabla_{x}^{2} u_{x}\right) d x=2 \int \operatorname{Ric}\left(\nabla_{t} \nabla_{x}^{2} u_{x}, \nabla_{x}^{2} u_{x}\right) d x \\
= & 2 \int \operatorname{Ric}\left(\nabla_{x} \nabla_{t} \nabla_{x} u_{x}, \nabla_{x}^{2} u_{x}\right) d x+2 \int \operatorname{Ric}\left(R\left(u_{t}, u_{x}\right) \nabla_{x} u_{x}, \nabla_{x}^{2} u_{x}\right) d x \\
= & 2 \int \operatorname{Ric}\left(\nabla_{x}^{3} u_{t}, \nabla_{x}^{2} u_{x}\right) d x+2 \int \operatorname{Ric}\left(\nabla_{x}\left(R\left(u_{t}, u_{x}\right) u_{x}\right), \nabla_{x}^{2} u_{x}\right) d x \\
& +2 \int \operatorname{Ric}\left(R\left(u_{t}, u_{x}\right) \nabla_{x} u_{x}, \nabla_{x}^{2} u_{x}\right) d x .
\end{aligned}
$$

Integrating by parts and substituting (1.4) yields

$$
\begin{aligned}
& \frac{d}{d t} \int \operatorname{Ric}\left(\nabla_{x}^{2} u_{x}, \nabla_{x}^{2} u_{x}\right) d x \\
= & -2 \int \operatorname{Ric}\left(\nabla_{x}^{5} u_{x}, \nabla_{x}^{2} u_{x}\right) d x-2 \rho \int \operatorname{Ric}\left(\operatorname{Ric}\left(u_{x}, u_{x}\right) u_{x}, \nabla_{x}^{5} u_{x}\right) \\
& -2 \int \operatorname{Ric}\left(R\left(\nabla_{x}^{2} u_{x}, u_{x}\right) u_{x}, \nabla_{x}^{3} u_{x}\right) d x \\
& +2 \int \operatorname{Ric}\left(R\left(\nabla_{x}^{2} u_{x}, u_{x}\right) \nabla_{x} u_{x}, \nabla_{x}^{2} u_{x}\right) d x .
\end{aligned}
$$


Here we used the property $R\left(\operatorname{Ric}\left(u_{x}, u_{x}\right) u_{x}, u_{x}\right) \equiv 0$. After integrating by parts, we could see that the firs term of (3.8) on the right vanishes, while the third term is equal to the fourth term, i.e.

$$
-2 \int \operatorname{Ric}\left(R\left(\nabla_{x}^{2} u_{x}, u_{x}\right) u_{x}, \nabla_{x}^{3} u_{x}\right) d x=2 \int \operatorname{Ric}\left(R\left(\nabla_{x}^{2} u_{x}, u_{x}\right) \nabla_{x} u_{x}, \nabla_{x}^{2} u_{x}\right) d x
$$

For the second term of (3.8), by the calculation of (2.13) we have

$$
\begin{aligned}
& -2 \rho \int \operatorname{Ric}\left(\nabla_{x}^{5} u_{x}, \operatorname{Ric}\left(u_{x}, u_{x}\right) u_{x}\right) \\
= & 20 \rho \int \operatorname{Ric}\left(\nabla_{x}^{2} u_{x}, \nabla_{x} u_{x}\right) \operatorname{Ric}\left(\nabla_{x}^{2} u_{x}, u_{x}\right) d x \\
& +10 \rho \int \operatorname{Ric}\left(\nabla_{x}^{2} u_{x}, \nabla_{x}^{2} u_{x}\right) \operatorname{Ric}\left(\nabla_{x} u_{x}, u_{x}\right) d x .
\end{aligned}
$$

Hence we have

$$
\begin{aligned}
& \frac{d}{d t} F_{1}=\frac{d}{d t} \int \operatorname{Ric}\left(\nabla_{x}^{2} u_{x}, \nabla_{x}^{2} u_{x}\right) d x \\
= & 20 \rho \int \operatorname{Ric}\left(\nabla_{x}^{2} u_{x}, \nabla_{x} u_{x}\right) \operatorname{Ric}\left(\nabla_{x}^{2} u_{x}, u_{x}\right) d x+10 \rho \int \operatorname{Ric}\left(\nabla_{x}^{2} u_{x}, \nabla_{x}^{2} u_{x}\right) \operatorname{Ric}\left(\nabla_{x} u_{x}, u_{x}\right) d x \\
& +4 \int \operatorname{Ric}\left(R\left(\nabla_{x}^{2} u_{x}, u_{x}\right) \nabla_{x} u_{x}, \nabla_{x}^{2} u_{x}\right) d x .
\end{aligned}
$$

For the second term of $E_{3}$, we calculate

$$
\begin{aligned}
& \frac{d}{d t} F_{2}=\frac{d}{d t} \int \operatorname{Ric}\left(\nabla_{x} u_{x}, u_{x}\right)^{2} d x \\
= & 2 \int \operatorname{Ric}\left(\nabla_{t} \nabla_{x} u_{x}, u_{x}\right) \operatorname{Ric}\left(\nabla_{x} u_{x}, u_{x}\right) d x+2 \int \operatorname{Ric}\left(\nabla_{x} u_{x}, \nabla_{t} u_{x}\right) \operatorname{Ric}\left(\nabla_{x} u_{x}, u_{x}\right) d x \\
= & 2 \int \operatorname{Ric}\left(\nabla_{x}^{2} u_{t}, u_{x}\right) \operatorname{Ric}\left(\nabla_{x} u_{x}, u_{x}\right) d x+2 \int \operatorname{Ric}\left(\nabla_{x} u_{x}, \nabla_{x} u_{t}\right) \operatorname{Ric}\left(\nabla_{x} u_{x}, u_{x}\right) d x \\
& +2 \int \operatorname{Ric}\left(\operatorname{R}\left(u_{t}, u_{x}\right) u_{x}, u_{x}\right) \operatorname{Ric}\left(\nabla_{x} u_{x}, u_{x}\right) d x .
\end{aligned}
$$

Note that the last term of (3.11) vanishes since

$$
\operatorname{Ric}\left(R\left(u_{t}, u_{x}\right) u_{x}, u_{x}\right)=\operatorname{Ric}\left(R\left(u_{x}, u_{x}\right) u_{x}, u_{t}\right)=0 .
$$

Substituting (1.4) into (3.11) we have:

$$
\begin{aligned}
& \frac{d}{d t} F_{2}=\frac{d}{d t} \int \operatorname{Ric}\left(\nabla_{x} u_{x}, u_{x}\right)^{2} d x \\
= & 2 \int \operatorname{Ric}\left(\nabla_{x}^{4} u_{x}, u_{x}\right) \operatorname{Ric}\left(\nabla_{x} u_{x}, u_{x}\right) d x+2 \int \operatorname{Ric}\left(\nabla_{x}^{3} u_{x}, \nabla_{x} u_{x}\right) \operatorname{Ric}\left(\nabla_{x} u_{x}, u_{x}\right) d x \\
& +2 \rho \int \operatorname{Ric}\left(\nabla_{x}^{2}\left(\operatorname{Ric}\left(u_{x}, u_{x}\right) u_{x}\right), u_{x}\right) \operatorname{Ric}\left(\nabla_{x} u_{x}, u_{x}\right) d x \\
& +2 \rho \int \operatorname{Ric}\left(\nabla_{x}\left(\operatorname{Ric}\left(u_{x}, u_{x}\right) u_{x}\right), \nabla_{x} u_{x}\right) \operatorname{Ric}\left(\nabla_{x} u_{x}, u_{x}\right) d x .
\end{aligned}
$$


After integrating by parts, we obtain

$$
\begin{aligned}
& 2 \int \operatorname{Ric}\left(\nabla_{x}^{4} u_{x}, u_{x}\right) \operatorname{Ric}\left(\nabla_{x} u_{x}, u_{x}\right) d x \\
= & -2 \int \operatorname{Ric}\left(\nabla_{x}^{3} u_{x}, \nabla_{x} u_{x}\right) \operatorname{Ric}\left(\nabla_{x} u_{x}, u_{x}\right) d x-2 \int \operatorname{Ric}\left(\nabla_{x}^{3} u_{x}, u_{x}\right) \operatorname{Ric}\left(\nabla_{x}^{2} u_{x}, u_{x}\right) d x \\
& -2 \int \operatorname{Ric}\left(\nabla_{x}^{3} u_{x}, u_{x}\right) \operatorname{Ric}\left(\nabla_{x} u_{x}, \nabla_{x} u_{x}\right) d x \\
= & 2 \int \operatorname{Ric}\left(\nabla_{x}^{2} u_{x}, \nabla_{x}^{2} u_{x}\right) \operatorname{Ric}\left(\nabla_{x} u_{x}, u_{x}\right) d x \\
& +8 \int \operatorname{Ric}\left(\nabla_{x}^{2} u_{x}, \nabla_{x} u_{x}\right) \operatorname{Ric}\left(\nabla_{x}^{2} u_{x}, u_{x}\right) d x \\
& +4 \int \operatorname{Ric}\left(\nabla_{x}^{2} u_{x}, \nabla_{x} u_{x}\right) \operatorname{Ric}\left(\nabla_{x} u_{x}, \nabla_{x} u_{x}\right) d x \\
= & 2 \int \operatorname{Ric}\left(\nabla_{x}^{2} u_{x}, \nabla_{x}^{2} u_{x}\right) \operatorname{Ric}\left(\nabla_{x} u_{x}, u_{x}\right) d x \\
& +8 \int \operatorname{Ric}\left(\nabla_{x}^{2} u_{x}, \nabla_{x} u_{x}\right) \operatorname{Ric}\left(\nabla_{x}^{2} u_{x}, u_{x}\right) d x
\end{aligned}
$$

Similarly, for the other three terms in (3.12), we could get the follow results:

$$
\begin{aligned}
& 2 \int \operatorname{Ric}\left(\nabla_{x}^{3} u_{x}, \nabla_{x} u_{x}\right) \operatorname{Ric}\left(\nabla_{x} u_{x}, u_{x}\right) d x \\
= & -2 \int \operatorname{Ric}\left(\nabla_{x}^{2} u_{x}, \nabla_{x}^{2} u_{x}\right) \operatorname{Ric}\left(\nabla_{x} u_{x}, u_{x}\right) d x-2 \int \operatorname{Ric}\left(\nabla_{x}^{2} u_{x}, \nabla_{x} u_{x}\right) \operatorname{Ric}\left(\nabla_{x}^{2} u_{x}, u_{x}\right) d x \\
& -2 \int \operatorname{Ric}\left(\nabla_{x}^{2} u_{x}, \nabla_{x} u_{x}\right) \operatorname{Ric}\left(\nabla_{x} u_{x}, \nabla_{x} u_{x}\right) d x \\
& 2 \rho \int \operatorname{Ric}\left(\nabla_{x}^{2}\left(\operatorname{Ric}\left(u_{x}, u_{x}\right) u_{x}\right), u_{x}\right) \operatorname{Ric}\left(\nabla_{x} u_{x}, u_{x}\right) d x \\
= & 2 \rho \int\left|\operatorname{Ric}\left(\nabla_{x} u_{x}, u_{x}\right)\right|^{3} d x-2 \rho \int \operatorname{Ric}\left(\nabla_{x} u_{x}, \nabla_{x} u_{x}\right) \operatorname{Ric}\left(\nabla_{x} u_{x}, u_{x}\right) \operatorname{Ric}\left(u_{x}, u_{x}\right) d x ;(3 .
\end{aligned}
$$

and

$$
\begin{aligned}
& 2 \rho \int \operatorname{Ric}\left(\nabla_{x}\left(\operatorname{Ric}\left(u_{x}, u_{x}\right) u_{x}\right), \nabla_{x} u_{x}\right) \operatorname{Ric}\left(\nabla_{x} u_{x}, u_{x}\right) d x \\
= & 4 \rho \int\left|\operatorname{Ric}\left(\nabla_{x} u_{x}, u_{x}\right)\right|^{3} d x+2 \rho \int \operatorname{Ric}\left(\nabla_{x} u_{x}, \nabla_{x} u_{x}\right) \operatorname{Ric}\left(\nabla_{x} u_{x}, u_{x}\right) \operatorname{Ric}\left(u_{x}, u_{x}\right) d x .(3.1
\end{aligned}
$$

In view of (3.12)-(3.16) we have

$$
\begin{aligned}
& \frac{d}{d t} F_{2}=\frac{d}{d t} \int \operatorname{Ric}\left(\nabla_{x} u_{x}, u_{x}\right)^{2} d x \\
= & 6 \int \operatorname{Ric}\left(\nabla_{x}^{2} u_{x}, \nabla_{x} u_{x}\right) \operatorname{Ric}\left(\nabla_{x}^{2} u_{x}, u_{x}\right) d x+6 \rho \int\left|\operatorname{Ric}\left(\nabla_{x} u_{x}, u_{x}\right)\right|^{3} d x .
\end{aligned}
$$

Next we compute $\frac{d}{d t} F_{3}$.

$$
\frac{d}{d t} F_{3}=\frac{d}{d t} \int \operatorname{Ric}\left(\nabla_{x} u_{x}, \nabla_{x} u_{x}\right) \operatorname{Ric}\left(u_{x}, u_{x}\right) d x
$$




$$
\begin{aligned}
= & 2 \int \operatorname{Ric}\left(\nabla_{t} \nabla_{x} u_{x}, \nabla_{x} u_{x}\right) \operatorname{Ric}\left(u_{x}, u_{x}\right) d x+2 \int \operatorname{Ric}\left(\nabla_{x} u_{x}, \nabla_{x} u_{x}\right) \operatorname{Ric}\left(\nabla_{t} u_{x}, u_{x}\right) d x \\
= & 2 \int \operatorname{Ric}\left(\nabla_{x}^{2} u_{t}, \nabla_{x} u_{x}\right) \operatorname{Ric}\left(u_{x}, u_{x}\right) d x+2 \int \operatorname{Ric}\left(\nabla_{x} u_{x}, \nabla_{x} u_{x}\right) \operatorname{Ric}\left(\nabla_{x} u_{t}, u_{x}\right) d x \\
& +2 \int \operatorname{Ric}\left(\operatorname{R}\left(u_{t}, u_{x}\right) u_{x}, \nabla_{x} u_{x}\right) \operatorname{Ric}\left(u_{x}, u_{x}\right) d x .
\end{aligned}
$$

Substituting (1.4) into (3.18) and integrating by parts yield

$$
\begin{aligned}
& \frac{d}{d t} F_{3}=\frac{d}{d t} \int \operatorname{Ric}\left(\nabla_{x} u_{x}, \nabla_{x} u_{x}\right) \operatorname{Ric}\left(u_{x}, u_{x}\right) d x \\
= & 6 \int \operatorname{Ric}\left(\nabla_{x}^{2} u_{x}, \nabla_{x}^{2} u_{x}\right) \operatorname{Ric}\left(\nabla_{x} u_{x}, u_{x}\right) d x-4 \rho \int\left|\operatorname{Ric}\left(\nabla_{x} u_{x}, u_{x}\right)\right|^{3} d x \\
& +10 \rho \int \operatorname{Ric}\left(\nabla_{x} u_{x}, \nabla_{x} u_{x}\right) \operatorname{Ric}\left(\nabla_{x} u_{x}, u_{x}\right) \operatorname{Ric}\left(u_{x}, u_{x}\right) d x \\
& -4 \rho \int \operatorname{Ric}\left(\nabla_{x} u_{x}, R\left(\nabla_{x} u_{x}, u_{x}\right) u_{x}\right) \operatorname{Ric}\left(\nabla_{x} u_{x}, u_{x}\right) d x .
\end{aligned}
$$

For the fourth term of $E_{3}(u)$, computing $\frac{d}{d t} F_{4}$ yields

$$
\begin{aligned}
& \frac{d}{d t} F_{4}=\frac{d}{d t} \int \operatorname{Ric}\left(\nabla_{x} u_{x}, R\left(\nabla_{x} u_{x}, u_{x}\right) u_{x}\right) d x \\
= & 2 \int \operatorname{Ric}\left(\nabla_{t} \nabla_{x} u_{x}, R\left(\nabla_{x} u_{x}, u_{x}\right) u_{x}\right) d x+2 \int \operatorname{Ric}\left(\nabla_{x} u_{x}, R\left(\nabla_{x} u_{x}, u_{x}\right) \nabla_{t} u_{x}\right) d x \\
= & 2 \int \operatorname{Ric}\left(\nabla_{x}^{2} u_{t}, R\left(\nabla_{x} u_{x}, u_{x}\right) u_{x}\right) d x+2 \int \operatorname{Ric}\left(R\left(u_{t}, u_{x}\right) u_{x}, R\left(\nabla_{x} u_{x}, u_{x}\right) u_{x}\right) d x \\
& +2 \int \operatorname{Ric}\left(\nabla_{x} u_{x}, R\left(\nabla_{x} u_{x}, u_{x}\right) \nabla_{x} u_{t}\right) d x \\
= & 2 \int \operatorname{Ric}\left(\nabla_{x}^{2} u_{t}, R\left(\nabla_{x} u_{x}, u_{x}\right) u_{x}\right) d x+2 \int \operatorname{Ric}\left(R\left(u_{t}, u_{x}\right) u_{x}, R\left(\nabla_{x} u_{x}, u_{x}\right) u_{x}\right) d x \\
& -2 \int \operatorname{Ric}\left(\nabla_{x}^{2} u_{x}, R\left(\nabla_{x} u_{x}, u_{x}\right) u_{t}\right) d x-2 \int \operatorname{Ric}\left(\nabla_{x} u_{x}, R\left(\nabla_{x}^{2} u_{x}, u_{x}\right) u_{t}\right) d x .
\end{aligned}
$$

We substitute (1.4) into each term of (3.20) on the right and obtain that:

for the first term of (3.20)

$$
\begin{aligned}
& 2 \int \operatorname{Ric}\left(\nabla_{x}^{2} u_{t}, R\left(\nabla_{x} u_{x}, u_{x}\right) u_{x}\right) d x \\
= & 2 \int \operatorname{Ric}\left(\nabla_{x}^{4} u_{x}, R\left(\nabla_{x} u_{x}, u_{x}\right) u_{x}\right) d x+2 \rho \int \operatorname{Ric}\left(\nabla_{x}^{2}\left(\operatorname{Ric}\left(u_{x}, u_{x}\right) u_{x}\right), R\left(\nabla_{x} u_{x}, u_{x}\right) u_{x}\right) d x \\
= & -2 \int \operatorname{Ric}\left(\nabla_{x}^{3} u_{x}, R\left(\nabla_{x}^{2} u_{x}, u_{x}\right) u_{x}\right) d x-2 \int \operatorname{Ric}\left(\nabla_{x}^{3} u_{x}, R\left(\nabla_{x} u_{x}, u_{x}\right) \nabla_{x} u_{x}\right) d x \\
& +2 \rho \int \operatorname{Ric}\left(\nabla_{x}^{2} u_{x}, R\left(\nabla_{x} u_{x}, u_{x}\right) u_{x}\right) \operatorname{Ric}\left(u_{x}, u_{x}\right) d x \\
& +8 \rho \int \operatorname{Ric}\left(\nabla_{x} u_{x}, R\left(\nabla_{x} u_{x}, u_{x}\right) u_{x}\right) \operatorname{Ric}\left(\nabla_{x} u_{x}, u_{x}\right) d x \\
= & 4 \int \operatorname{Ric}\left(\nabla_{x}^{2} u_{x}, R\left(\nabla_{x}^{2} u_{x}, u_{x}\right) \nabla_{x} u_{x}\right) d x+6 \rho \int \operatorname{Ric}\left(\nabla_{x} u_{x}, R\left(\nabla_{x} u_{x}, u_{x}\right) u_{x}\right) \operatorname{Ric}\left(\nabla_{x} u_{x}, u_{x}\right) d x .
\end{aligned}
$$


For the last three terms of (3.20) we have

$$
\begin{aligned}
& 2 \int \operatorname{Ric}\left(R\left(u_{t}, u_{x}\right) u_{x}, R\left(\nabla_{x} u_{x}, u_{x}\right) u_{x}\right) d x-2 \int \operatorname{Ric}\left(\nabla_{x}^{2} u_{x}, R\left(\nabla_{x} u_{x}, u_{x}\right) u_{t}\right) d x \\
& -2 \int \operatorname{Ric}\left(\nabla_{x} u_{x}, R\left(\nabla_{x}^{2} u_{x}, u_{x}\right) u_{t}\right) d x \\
= & 2 \int \operatorname{Ric}\left(R\left(\nabla_{x}^{2} u_{x}, u_{x}\right) u_{x}, R\left(\nabla_{x} u_{x}, u_{x}\right) u_{x}\right) d x-2 \int \operatorname{Ric}\left(\nabla_{x} u_{x}, R\left(\nabla_{x}^{2} u_{x}, u_{x}\right) \nabla_{x}^{2} u_{x}\right) d x \\
& -2 \rho \int \operatorname{Ric}\left(\nabla_{x}^{2} u_{x}, R\left(\nabla_{x} u_{x}, u_{x}\right) u_{x}\right) \operatorname{Ric}\left(u_{x}, u_{x}\right) d x \\
& -2 \rho \int \operatorname{Ric}\left(\nabla_{x} u_{x}, R\left(\nabla_{x}^{2} u_{x}, u_{x}\right) u_{x}\right) \operatorname{Ric}\left(u_{x}, u_{x}\right) d x \\
= & 2 \int \operatorname{Ric}\left(\nabla_{x}^{2} u_{x}, R\left(\nabla_{x}^{2} u_{x}, u_{x}\right) \nabla_{x} u_{x}\right) d x-2 \int \operatorname{Ric}\left(R\left(\nabla_{x} u_{x}, u_{x}\right) \nabla_{x} u_{x}, R\left(\nabla_{x} u_{x}, u_{x}\right) u_{x}\right) d x \\
& +4 \rho \int \operatorname{Ric}\left(\nabla_{x} u_{x}, R\left(\nabla_{x} u_{x}, u_{x}\right) u_{x}\right) \operatorname{Ric}\left(\nabla_{x} u_{x}, u_{x}\right) d x .
\end{aligned}
$$

Combining (3.20), (3.21) and (3.22) yields

$$
\begin{aligned}
& \frac{d}{d t} F_{4}=\frac{d}{d t} \int \operatorname{Ric}\left(\nabla_{x} u_{x}, R\left(\nabla_{x} u_{x}, u_{x}\right) u_{x}\right) d x \\
= & 6 \int \operatorname{Ric}\left(\nabla_{x}^{2} u_{x}, R\left(\nabla_{x}^{2} u_{x}, u_{x}\right) \nabla_{x} u_{x}\right) d x-2 \int \operatorname{Ric}\left(R\left(\nabla_{x} u_{x}, u_{x}\right) \nabla_{x} u_{x}, R\left(\nabla_{x} u_{x}, u_{x}\right) u_{x}\right) d x \\
& +10 \rho \int \operatorname{Ric}\left(\nabla_{x} u_{x}, R\left(\nabla_{x} u_{x}, u_{x}\right) u_{x}\right) \operatorname{Ric}\left(\nabla_{x} u_{x}, u_{x}\right) d x .
\end{aligned}
$$

Hence by (3.10), (3.17), (3.19) and (3.23) we obtain

$$
\begin{aligned}
& \quad \frac{d}{d t} E_{3}(u)=\frac{d}{d t}\left(\sum_{i=1}^{4} A_{i} F_{i}\right) \\
& =\quad\left(20 \rho A_{1}+6 A_{2}\right) \int \operatorname{Ric}\left(\nabla_{x}^{2} u_{x}, \nabla_{x} u_{x}\right) \operatorname{Ric}\left(\nabla_{x}^{2} u_{x}, u_{x}\right) d x \\
& \quad+\left(10 \rho A_{1}+6 A_{3}\right) \int \operatorname{Ric}\left(\nabla_{x}^{2} u_{x}, \nabla_{x}^{2} u_{x}\right) \operatorname{Ric}\left(\nabla_{x} u_{x}, u_{x}\right) d x \\
& +\left(4 A_{1}+6 A_{4}\right) \int \operatorname{Ric}\left(\nabla_{x}^{2} u_{x}, R\left(\nabla_{x}^{2} u_{x}, u_{x}\right) \nabla_{x} u_{x}\right) d x \\
& +2 \rho\left(5 A_{4}-2 A_{3}\right) \int \operatorname{Ric}\left(\nabla_{x} u_{x}, R\left(\nabla_{x} u_{x}, u_{x}\right) u_{x}\right) \operatorname{Ric}\left(\nabla_{x} u_{x}, u_{x}\right) d x \\
& +2 \rho\left(3 A_{2}-2 A_{3}\right) \int\left|\operatorname{Ric}\left(\nabla_{x} u_{x}, u_{x}\right)\right|^{3} d x \\
& +10 \rho A_{3} \int \operatorname{Ric}\left(\nabla_{x} u_{x}, \nabla_{x} u_{x}\right) \operatorname{Ric}\left(\nabla_{x} u_{x}, u_{x}\right) \operatorname{Ric}\left(u_{x}, u_{x}\right) d x \\
& \quad-2 A_{4} \int \operatorname{Ric}\left(R\left(\nabla_{x} u_{x}, u_{x}\right) \nabla_{x} u_{x}, R\left(\nabla_{x} u_{x}, u_{x}\right) u_{x}\right) d x .
\end{aligned}
$$


Since $A_{1}=6, A_{2}=-20 \rho, A_{3}=-10 \rho, A_{4}=-4$, it easy to see that the first three terms of (3.24) vanish. Hence

$$
\begin{aligned}
\frac{d}{d t} E_{3}(u)= & -80 \rho^{2} \int\left|\operatorname{Ric}\left(\nabla_{x} u_{x}, u_{x}\right)\right|^{3} d x \\
& 40 \rho(-1+\rho) \int \operatorname{Ric}\left(\nabla_{x} u_{x}, R\left(\nabla_{x} u_{x}, u_{x}\right) u_{x}\right) \operatorname{Ric}\left(\nabla_{x} u_{x}, u_{x}\right) d x \\
& -100 \rho^{2} \int \operatorname{Ric}\left(\nabla_{x} u_{x}, \nabla_{x} u_{x}\right) \operatorname{Ric}\left(\nabla_{x} u_{x}, u_{x}\right) \operatorname{Ric}\left(u_{x}, u_{x}\right) d x \\
& +8 \int \operatorname{Ric}\left(R\left(\nabla_{x} u_{x}, u_{x}\right) \nabla_{x} u_{x}, R\left(\nabla_{x} u_{x}, u_{x}\right) u_{x}\right) d x \\
\leq & C(N, \rho) \int\left|\nabla_{x} u_{x}\right|^{3}\left|u_{x}\right|^{3} d x \\
\leq & C(N, \rho)\left\|u_{x}\right\|_{L^{\infty}}^{3} \int\left|\nabla_{x} u_{x}\right|^{3} d x .
\end{aligned}
$$

Using the following interpolation inequalities (see [8] for details)

$$
\begin{aligned}
\left\|u_{x}\right\|_{L^{\infty}} & \leq C(N)\left(\left\|\nabla_{x} u_{x}\right\|_{L^{2}}^{2}+\left\|u_{x}\right\|_{L^{2}}^{2}\right)^{\frac{1}{4}}\left\|u_{x}\right\|_{L^{2}}^{\frac{1}{2}} \\
& \leq C\left(N,\left\|\nabla_{x} u_{x}\right\|_{L^{2}}, E_{1}\left(u_{0}\right)\right) ; \\
\left\|\nabla_{x} u_{x}\right\|_{L^{3}}^{3} & \leq C(N)\left(\left\|\nabla_{x}^{2} u_{x}\right\|_{L^{2}}^{2}+\left\|\nabla_{x} u_{x}\right\|_{L^{2}}^{2}\right)^{\frac{1}{4}}\left\|\nabla_{x} u_{x}\right\|_{L^{2}}^{\frac{5}{2}} \\
& \leq C\left(N,\left\|\nabla_{x} u_{x}\right\|_{L^{2}}\right)\left(1+\left\|\nabla_{x}^{2} u_{x}\right\|_{L^{2}}^{2}\right),
\end{aligned}
$$

we have

$$
\frac{d E_{3}}{d t} \leq C\left(1+\int\left|\nabla_{x}^{2} u_{x}\right|^{2} d x\right)
$$

where $C=C\left(N,\left\|\nabla_{x} u_{x}\right\|_{L^{2}}, E_{1}\left(u_{0}\right), \rho\right)>0$ only depends on $N, E_{1}\left(u_{0}\right)$ and $\left\|\nabla_{x} u_{x}\right\|_{L^{2}}$. By the assumption, if Ric $\geq \lambda>0$, we have

$$
\int\left|\nabla_{x}^{2} u_{x}\right|^{2} d x \leq \frac{1}{\lambda} \int \operatorname{Ric}\left(\nabla_{x}^{2} u_{x}, \nabla_{x}^{2} u_{x}\right) d x \leq C_{1} E_{3}+C_{2},
$$

where $C_{1}, C_{2}$ only depend on $N, \lambda$ and $\left\|\nabla_{x} u_{x}\right\|_{L^{2}}$. This together with (3.26) yields

$$
\frac{d E_{3}}{d t} \leq C\left(1+E_{3}\right)
$$

where $C=C\left(N, \lambda,\left\|\nabla_{x} u_{x}\right\|_{L^{2}}, E_{1}\left(u_{0}\right)\right)>0$ only depends on $N, \lambda, E_{1}\left(u_{0}\right)$ and $\left\|\nabla_{x} u_{x}\right\|_{L^{2}}$.

It is easy to check that for the case the Ricci curvature on $N$ has a negative upper bound $-\lambda<0$, we could also get the result via a same argument. Hence we complete the proof of the lemma.

\section{Global existence}

In this section we derive the global existence of the Cauchy problem (1.4) and prove Theorem 1.3. Since $u_{0} \in H^{4}\left(S^{1}, N\right)$, we can always choose a sequence of smooth maps $u_{0 i} \in C^{\infty}\left(S^{1}, N\right)$ such that, as $i \rightarrow \infty$,

$$
\left\|u_{0 i}-u_{0}\right\|_{H^{4}} \rightarrow 0
$$


By the arguments in Theorem 1.2, we get that the Cauchy problem (1.4) with the initial map $u_{0 i}$ admits a unique smooth local solution $u^{i}$ such that

$$
\left.u^{i} \in C\left(\left[0, T\left(N,\left\|u_{0 i}\right\|_{H^{4}}\right)\right], H^{k}\left(S^{1}, N\right)\right)\right)
$$

for any $k \geq 4$. Obviously, it is easy to see that $T\left(N,\left\|u_{0 i}\right\|_{H^{4}}\right)$ have a uniform lower bound. Hence, letting $i \rightarrow \infty$, we obtain the local solution to the Cauchy problem of the new geometric flow with the initial map $u_{0} \in H^{4}\left(S^{1}, N\right)$. Thus, to prove Theorem 1.3, we only need to consider the case $u_{0}$ is a smooth map from $S^{1}$ into $N$.

Let $u$ be the local smooth solution of (1.4) which exists on the maximal time interval $[0, T)$. We only discuss the case that $T<\infty$ and we assume the Ricci curvature on $N$ has a positive lower bound $\lambda>0$.

From Lemma 3.1, we know that the energy is bounded by $E_{1}\left(u_{0}\right)$ because

$$
0 \leq \int\left|u_{x}\right|^{2} d x \leq \frac{1}{\lambda} E_{1}(u(t))=\frac{1}{\lambda} E_{1}\left(u_{0}\right), \quad \text { for any } \quad t \in[0, T) .
$$

Moreover, from Lemma 3.2 we know that $E_{2}$ is preserved, that is

$$
E_{2}(u)=\int \operatorname{Ric}\left(\nabla_{x} u_{x}, \nabla_{x} u_{x}\right) d x-\frac{\rho}{2} \int \operatorname{Ric}\left(u_{x}, u_{x}\right)^{2} d x=E_{2}\left(u_{0}\right)
$$

Thus we have

$$
\begin{aligned}
\left\|\nabla_{x} u_{x}\right\|_{L^{2}}^{2} & \leq \frac{1}{\lambda} \int \operatorname{Ric}\left(\nabla_{x} u_{x}, \nabla_{x} u_{x}\right) d x \operatorname{Ric} \\
& =\frac{1}{\lambda}\left(E_{3}\left(u_{0}\right)+\frac{\rho}{2} \int \operatorname{Ric}\left(u_{x}, u_{x}\right)^{2} d x\right) \\
& \leq C(N, \lambda, \rho)\left(E_{3}\left(u_{0}\right)+\int\left|u_{x}\right|^{4} d x\right) \\
& \leq C\left(N, E_{1}\left(u_{0}\right), \lambda, E_{3}\left(u_{0}\right), \rho\right),
\end{aligned}
$$

note that here we used the interpolation inequality

$$
\begin{aligned}
\left\|u_{x}\right\|_{L^{4}}^{4} & \leq\left(\left\|\nabla_{x} u_{x}\right\|_{L^{2}}^{2}+\left\|u_{x}\right\|_{L^{2}}^{2}\right)^{\frac{1}{2}}\left\|u_{x}\right\|_{L^{2}}^{3} \\
& \leq \frac{1}{2}\left\|\nabla_{x} u_{x}\right\|_{L^{2}}^{2}+C\left(E_{1}\left(u_{0}\right), \lambda\right) .
\end{aligned}
$$

Then, (4.1) together with Lemma 3.3 yields

$$
\frac{d E_{3}}{d t} \leq C\left(N, E_{1}\left(u_{0}\right), E_{2}\left(u_{0}\right), \rho\right)\left(1+E_{3}\right) .
$$

By Gronwall inequality, we get that $E_{3}(u(t))$ is uniformly bounded on $[0, T)$. Hence, we obtain

$$
\begin{aligned}
6 \lambda \int\left\|\nabla_{x}^{2} u_{x}\right\|^{2} d x & \leq 6 \int \operatorname{Ric}\left(\nabla_{x}^{2} u_{x}, \nabla_{x}^{2} u_{x}\right) d x \\
& =E_{3}(u)+10 \int \operatorname{Ric}\left(\nabla_{x} u_{x}, u_{x}\right)^{2} d x
\end{aligned}
$$




$$
\begin{aligned}
& +5 \int \operatorname{Ric}\left(\nabla_{x} u_{x}, \nabla_{x} u_{x}\right) \operatorname{Ric}\left(u_{x}, u_{x}\right) d x \\
& +4 \int \operatorname{Ric}\left(\nabla_{x} u_{x}, R\left(\nabla_{x} u_{x}, u_{x}\right) u_{x}\right) d x \\
\leq & C\left(N, \lambda, E_{1}\left(u_{0}\right), E_{2}\left(u_{0}\right), E_{3}\left(u_{0}\right), \rho\right)+C(N)\left\|u_{x}\right\|_{L^{\infty}}^{2}\left\|\nabla_{x} u_{x}\right\|_{L^{2}}^{2} .
\end{aligned}
$$

In view of (3.25), (4.1) and the boundedness of $E_{3}$, we see that $\left\|\nabla_{x}^{2} u_{x}\right\|_{L^{2}}$ is uniformly bounded on $[0, T)$. Hence we have

$$
\sup _{t \in[0, T)}\left\|u_{x}\right\|_{H^{2}} \leq C\left(N, \lambda, E_{1}\left(u_{0}\right), E_{2}\left(u_{0}\right), E_{3}\left(u_{0}\right), \rho\right) .
$$

It follows from the proof of Lemma 2.2 that for $m>2$

$$
\sup _{t \in[0, T)}\left\|\nabla_{x}^{m} u_{x}\right\|_{L^{2}} \leq C\left(N,\left\|u_{0 x}\right\|_{L^{2}},\left\|\nabla_{x} u_{0 x}\right\|_{L^{2}},\left\|\nabla_{x}^{2} u_{0 x}\right\|_{L^{2}}, \cdots,\left\|\nabla_{x}^{m} u_{0 x}\right\|_{L^{2}}, \rho\right) .
$$

Thus, if $T$ is finite, we can find a time-local solution $u_{1}$ of (1.4) and $u_{1}$ satisfies the initial value condition

$$
u_{1}(x, T-\epsilon)=u(x, T-\epsilon),
$$

where $0<\epsilon<T$ is a small number. Then by the local existence theorem, $u_{1}$ exists on the time interval $(T-\epsilon, T-\epsilon+\eta)$ for some constant $\eta>0$. The uniform bounds on $\left\|u_{x}\right\|_{H^{2}}$ and $\left\|\nabla_{x}^{m} u_{x}\right\|_{L^{2}}$ (for all $m>2$ ) implies that $\eta$ is independent of $\epsilon$. Thus, by choosing $\epsilon$ sufficiently small, we have

$$
T_{1}=T-\epsilon+\eta>T \text {. }
$$

By the uniqueness result, we have that $u_{1}(x, t)=u(x, t)$ for all $t \in\left[T-\epsilon, T_{1}\right)$. Thus we get a solution of the Cauchy problem (1.4) on the time interval $\left[0, T_{1}\right)$, which contradicts the maximality of $T$.

For the case that the Ricci curvature on $N$ has a negative upper bound $-\lambda<0$, we still could obtain the results via the same arguments and we omit the details. Thus we complete the proof of Theorem 1.3.

\section{References}

[1] I. Bejenaru, A. D. Ionescu, C. E. Kenig, D. Tataru; Global Schrödinger maps, preprint.

[2] N. H. Chang, J. Shatah, K. Uhlenbeck; Schrödinger maps, Commun. Pure Appl. Math. 53(2000), 590-602.

[3] J. Colliander, M. Keel, G. Staffilani, H. Takaoka, and T. Tao; Sharp global well-posedness for KdV and modified $K d V$ on $\mathbb{R}$ and $\mathbb{T}$, J. Amer. Math. Soc. 16(2003), 705-749.

[4] E. Datet, M. Jimbo, M. Kashiwara and T. Miwa; Landau-Lifshitz equation: solitons, quasi-periodic solutions and infinite-dimensional Lie algebras, Phys. A: Math. Gen. 16 (1983) 221-236.

[5] Q. Ding, Y. D. Wang; Geometric KdV flows, motions of curves and the third-order system of the AKNS hierarchy, Internat. J. Math. 22 (2011), no. 7, 1013C1029.

[6] W. Y. Ding; On the Schrödinger flows, Proc. ICM Beijing 2002, 283-292. 
[7] W. Y. Ding, Y. D. Wang; Schrödinger flows of maps into symplectic manifolds, Sci. China A41(1998), 746-755.

[8] W. Y. Ding, Y. D. Wang; Local Schrödinger flow into Kähler manifolds, Sci. China A44(2001), 1446-1464.

[9] A. Friedman, Partial differential equations of parabolic type, Prentice-Hall, Inc., Englewood Cliffs, N.J. (1964).

[10] Y. Fukumoto, T. Miyazaki; Three-dimensional distortions of a vortex filament with axial velocity, J. Fluid Mech. 222(1991), 369-416.

[11] V. Gerdjikov, N. A. Kostov; Reductions of multicomponent mKdV equations on symmetric spaces of DIII-type, SIGMA 4(2008), 29-58.

[12] I. Golubchik, V. Sokolov; Multicomponent generalization of the hierarchy of the Landau-Lifshitz equation, Theor. and Math. Phys., 124(2000), no. 1, 909-917.

[13] H. Hasimoto; A soliton on a vortex filament, J. Fluid mech. 51(1972), 477-485.

[14] S. Helgason; Differential Geometry, Lie Groups, and Symmetric Spaces, AMS, Providence, Rhode Island.

[15] R. Hirota; Exact envelope-soliton solutions of a nonlinear wave equation, J. Math. Phys. 14(1973), 805-809.

[16] A. Hasegawa, Y. Kodama; Nonlinear pulse propagation in a monomode dielectric guide, IEEE J. Quantum Elec. 23(1987), 510-524.

[17] A. Meshkov, V. Sokolov; Integrable evolution equations on the $n$-dimensional sphere, Comm Math Phys, 232(2002), 1-18.

[18] K. Moffatt, L. Ricca; Interpretation of invariants of the Betchov-Da rios equation and of the euler equations, The Global Geometry of Turbulence, Plenum Press, New York (1991).

[19] T. Nishiyama, A. Tani; Initial and initial-boundary value problems for a vortex filament with or without axial flow, SIAM J. Math. Anal. 27(1996), 1015-1023.

[20] A. Tani, T. Nishiyama; Solvability of equations for motion of a vortex filament with or without axial flow, Publ. Res. Inst. Math. Sci. 33(1997), 509-526.

[21] E. Onodera; A third-order dispersive flow for closed curves into Kähler manifolds, J. Geom. Anal. 18(2008), 889-918.

[22] P. Petersen; Riemannian Geometry, New York: Springer Verlag(1998).

[23] R. Ricca; Rediscovery of Da Rios equations, Nature 352, 561-562(1991).

[24] I. Rodnianski, Y. A. Rubinstein, G. Staffilani; On the global well-posedness of the one-dimensional Schrodinger map flow, Analysis and PDE, 2(2009), 187-209.

[25] C. Song; The KdV curve and Schrödinger-Airy curve, Proc. Amer. Math. Soc., 140 (2012), no. 2, 635-644.

[26] C. Song, J. Yu; The Cauchy problem of generalized Landau-Lifshitz equation into $S^{n}$, Sci. China Math. 56 (2013), no. 2, 283-300.

[27] X. W. Sun, Y. D. Wang; KdV Geometric Flows on Kähler Manifolds, Inter. J. Math., 22(2011), $1-62$.

[28] X. W. Sun, Y. D. Wang; Geometric Schrödinger-Airy Flows on Kähler Manifolds, Acta Math. Sinica, English series, 29(2013), no.2, 209-240.

[29] T. Tsuchida, M.Wadati; The coupled modified Korteweg-de Vries equations, J. Phys. Soc. Jpn. 67(1998), 1175-1187.

[30] T. Tsuchida; New reductions of integrable matrix partial differential equations: Sp(m)-invariant systems, J. Math. Phys. 51 (2010), no.5, 053511, 27 pp. 
[31] M. E. Taylor; Partial Differential Equations III: Nonlinear Equations, New York: Springer Ver$\operatorname{lag}(1997)$.

[32] A.P. Veselov; Finite-gap potentials and integrable systems on the sphere with a quadratic potential, Funct. Anal. and Appl. 14(1980), no.1, 48-50.

[33] B. Wang, L. Han, C. Huang; Global well-posedness and scattering for the derivative nonlinear Schrödinger equation with small rough data, Ann. Inst. H. Poincar Anal. Non Linéaire 26(2009), no.6, 2253-2281.

Xiaowei Sun

School of Statistics and Mathematics,

Central University of Finance and Economics

Beijing 100081, P.R. China.

Email:sunxw@cufe.edu.cn

Youde Wang

Academy of Mathematics and Systems Science

Chinese Academy of Sciences,

Beijing 100190, P.R. China.

Email: wyd@math.ac.cn 